\title{
Bridging the gap among actor-sensor- actor communication through load balancing multi-path routing
}

\author{
Jun Long, Chao Gao, Sheng He, Xiao Liu and Anfeng Liu*
}

\begin{abstract}
Wireless sensor actor networks (WSANs) consist of a large amount of sensor nodes with low cost and little actor nodes with better processing capabilities. The actor nodes tend to get partitioned due to low actor density in case of economic considerations. So, the communication among actors requires sensor nodes to relay his data to the destination actor which lead to the bottleneck in communication. A high-throughput disjoint multi-path (HTDM) routing scheme is proposed in this paper which allows actor simultaneously forwards data through multiple disjoint paths to achieve high throughput. HTDM routing scheme can quickly establish multiple disjoint routing paths, which can increase the throughput among actors a lot, and the routing path can be adjusted dynamically according to the energy status of their nodes, which can achieve load balancing. Simulation results show that, compared with related routing schemes, HTDM routing scheme can achieve higher throughput performance and distribute transmission loads more evenly to most of the nodes in the network.
\end{abstract}

Keywords: Wireless sensor networks, Throughput, Disjoint multi-path routing, Network lifetime, Load balancing

\section{Introduction}

Wireless sensor actor networks (WSANs) are composed of a lot of sensor nodes and the amount of actor nodes $[1,2]$. WSANs and other wireless network can monitor the environment through sensor nodes, then send these information to corresponding actor nodes; actor nodes can judge, analyze and take action on detection an event [3-14]. This feature of WSANs also makes it more widely used in environmental monitoring, health monitoring, smart home, animal positioning and tracking, intelligent transportation, precision agriculture, and other civilian areas in the future [15-23]. The wireless-enabled actor nodes are equipped with better processing capabilities, higher transmission powers, and longer battery life, and its resource is rich $[1,2]$. It is common in WSANs that a large number of sensor nodes [23-27] and a relatively fewer number of actor nodes are

\footnotetext{
* Correspondence: afengliu@mail.csu.edu.cn

School of Information Science and Engineering, Central South University, ChangSha 410083, China
}

deployed in the terrain under monitoring [1-3]. The number of actor nodes is less due to they are related to the cost. Effective sensor-to-actor communication (SAc) and actor-to-actor communication (AAc) are two important issues in WSANs. If the deployment of actor nodes can connect with each other, AAc is not a serious problem [1-3]. But if the number of actor nodes deployed in the terrain is not large enough, this causes the actor network topology becomes sparse and achieving effective AAc becomes an important problem [1-3]. In order to heal the partitions in the actor network, using the sensor nodes to support the communication among actors could be a reasonable solution [28-35]. When the actor cannot communicate with other actors through AAc, it could forward its data to the sensor nodes, and uses the sensor nodes to relay its data to the target actor [35-40]. Guo et al. [8] call this transmission pattern actor-sensoractor communication (ASAc).

But due to the huge difference of communication capacity between actors and sensor nodes, the routing transmission of ASAc becomes the communication

\section{望 Springer}


bottleneck of the whole network. The system not only exist the mismatch problem for the communication rate in ASAc, but also the unbalance problems of energy consumption in previous schemes [22]. In previous researches, the communication among partition actors uses the shortest routing method. There exist unbalanced problems of energy consumption: (1) the nodes among partition actors in the shortest path are often chosen as routing, and the nodes in other area are seldom selected as routing [22], which cause unbalanced energy consumption; (2) overall, sensor nodes in the center area of network often become routing path node of multiple pair of partition actors, thus, its energy consumption is higher. The probability of those nodes, which is at the edge area of the network, selected as routing is small, this causes the nodes at the central region of network first died and the peripheral area of network left much energy, which affecting the network lifetime [22, 41]. Therefore, it is important to balance energy consumption to alleviate or eliminate communication bottleneck among partition actors in WSANs.

In order to overcome the shortcomings of ASAc for WSANs in previously researchers, a high-throughput disjoint multi-path (HTDM) routing scheme is proposed to solve this issue. The major contributions of this paper are the following:

(1) A HTDM routing scheme is proposed in this paper. HTDM routing is different from previous researches in two main features. (a) Several disjoint routing is built simultaneously in HTDM routing scheme, which can be largely improve communication throughput of partition actors due to data are transmitted through multiple paths simultaneously, thus largely smooth communication capabilities between actors and sensor nodes. The network delay can be reduced. (b) The difference from multi-path routing of the previous studies is the real non-interfering multi-path routing is established from the source node to the destination node in HTDM routing scheme, which can greatly improve the throughput of the system. In previous schemes, multiple routing have the same source or destination node, therefore, the data cannot be transmitted simultaneously, which did not realize multi-path routing [42].

(2) HTDM routing scheme has a better capacity for balancing energy consumption and prolonging network life. In HTDM scheme, the more left energy of the routing (though the path may be longer) is, the bigger the probability of routing selected as routing path is, so as to make the network energy consumption balanced as far as possible, which prolong network lifetime. We regard the HTDM problem as an optimization problem and propose a new routing path optimization approach to fix it.

(3) Through our extensive theoretical analysis and simulation study, we demonstrate that for HTDM routing scheme, both throughput and lifetime can be improved simultaneously. Comparing with the previous actor-sensor-actor communication (ASAc) scheme, HTDM routing scheme has three advantages. Firstly, it takes full advantage of the energy, and the energy utilization ratio of network can be improved largely, so it can improve the network lifetime. Secondly, the throughput can be improved several times. Thirdly, the HTDM routing scheme can significantly smooth communication capabilities of the capabilities between actors and sensor nodes, so the network delay is reduced.

The rest of this paper is organized as follows: in Section 2, the related works are reviewed. The system model and problem statement are described in Section 3. In Section 4, the details of disjoint multi-path for highthroughput routing scheme are presented. Experimental results and comparison are given in Section 5. We conclude in Section 6.

\section{Related work}

The core problems of wireless sensor actor networks (WSANs) are to deploy network using the most economical style and to monitor the network with low latency, energy efficient, high throughput through the coordination between sensor nodes and actors. There are a lot of issues need to be studied on WSANs [1, 2, 21, 43-46].

Melodia et al. [47] presented a coordination framework for WSANs and discussed the sensor-actor and actor-actor coordination problems. For sensor-actor communication model, sensor nodes are partitioned to different sets associated with one or more actors based on event-driven partitioning. They formulated it as an integer linear problem (ILP). When the event occurs, sensor nodes send the sensed information to the actor in their set, then many actors in different sets communicate with each other to reach the purpose of coordination communication of the whole network. For actoractor communication model, Melodia et al. [47] regard coordination problem formulate among actors as a mixed integer non-linear program under condition that the energy needed for actor mobility can be conserved and fulfill the deadline and resource constraints of events.

The use of directional antenna at sink node is proposed in Ref. [47]. The most similar scheme is energy 
efficient directional routing (EEDR) protocol which is proposed in Ref. [1]. Network communication of WSANs can be divided into the following categories by Selvaradjou et al. [1]: (a) the sensor-actor communication. This kind of communication is similar to the communication between sensor node and sink node. Common sensor node route data to actor using shortrange communication channel through multiple hop method. (b) The actor-actor communication in a set. Because actor nodes have more resources and stronger communication ability, thus, the actor-actor communication within a set adopts the long-range communication channel with farther communication distance and higher throughput. (c) The actor-actor communication in different sets. This type of communication requires sensor nodes to relay data. EEDR protocol make data reach sensor nodes nearest to the destination actor using the directional broadcasts way, which resulting in the lower end-to-end latency and lower energy consumption. But the communication difference between actor and sensor node is huge, EEDR protocol can only realize the actor-actor communication, but not give the method for how to make up the bottleneck problem.

In WSANs, routing policy for improving energy efficient is an important issue to study. It is different from the wireless sensor network because the WSANs have multiple actors, each actor node is equivalent to a sink in the WSNs. Therefore, sensor node sends data to an actor, which is similar to send data to virtual sink. Wan et al. [48] studied how does dispatch, the traffic generated by sensor nodes to the physical sink via a set of virtual sinks in case of congestion notification. Zhou et al. [2] proposed a power aware routing protocol named power-speed that performs energy-efficient and timely reporting of events from sensor nodes to any of the actor nearest to actors.

Unlike WSNs, in WSANs, actor can move to perform a particular task; this task can be a data collection, or the event handling. So, the efficient events detecting in terms of limit time and energy consumption of actors' mobility in WSANs is another important issue.

ORACLE approach is proposed to make actors predict events before sensors detection and migrate to the areas where the event may occur [49]. Then, Kaoru et al. propose an event prediction scheme to predict an event from collected sensory data by utilizing the maximum likelihood estimation. Based on the perception, they design a control policy of actor's mobility pattern with Markov decision process.

The previous routing approaches which are mainly focused on creating route path to transmit data. The route path can be divided two parts: one is the routing of actor. In order to make effective energy consumption, the shortest length of routing path, and the lowest routing time, the main problem is how to plan routing path of actor when an event occurs. Those researches always adopt linear programming and prediction method [49] to achieve the above objectives. But the shortages of those schemes are: because the occurrence of the event is unpredictable, if the scheme can predict the location where the event occurs, actor can move to the area where the event occurs using the method of prediction to deal with the problem quickly. But if it does not, it will cost more to solve this problem. Another is actorsensor-actor communication (ASAc) which is same as our research. In the main task of this scheme is that detecting sensed areas need to be completed by actor with deployed sensor nodes. In WSANs, the difference of building routing between actor-sensor communication and wireless sensor networks (WSNs) is the routing is generally stable in WSNs, the destination node of routing path is always sink. But in WSANs, the routing is dynamic. Because actors often move, which resulting in network topology changes frequently, this leads to its routing requires to be adjusted constantly. Thus, a better robust are needed for establishing routing mechanism to adapt to this change. At present, routing mechanism is still in the primary stage in WSANs, the main goal is to establish communication routing, and the routing quality is less considered $[1,21,47,50]$.

\section{The system model and problem statement}

\subsection{The system model}

(1) According to Ref. [1, 21], a large number of sensor nodes and several actors are randomly deployed in the WSAN. The maximum transmission ranges of actors and sensor nodes are represented by $r_{\mathrm{a}}$ and $r_{\mathrm{s}}$, respectively. In practice, the transmission range of actors is always greater than that of sensor nodes'. The neighboring distance is the maximal reachable distance with the transmission power for neshows the process of establishing multiighboring sensors. For a given sensor/actors, the sensors/ actors within its neighboring distance are its "neighboring sensors/actors" or "neighbors". Each sensor can be aware of the current energy level of its neighbors by anticipating and/or eavesdropping for data from the neighbors. To save the cost of network, HTDM scheme assumes that the sensor node has no GPS positioning devices, so HTDM scheme has more extensive applicability. But the actor nodes with GPS positioning devices, and they are not energy constrained. All sensor nodes have limited energy and the actors are of unlimited energy. 
(2) Communication rate among actor nodes is $v_{\mathrm{a}}$, communication rate among sensor nodes is $v_{\mathrm{s}}$, in generally, $v_{\mathrm{a}}>>v_{s}$. The same as Ref. [21], all the actors which can communicate with each other directly through actor node are called an actor set (AS). In an actor set, the communication among actors (AAc) adopts long-range communication channel. The communication among sensors adopts short-range communication channel. Actor nodes communicate with each other over a long-range communication channel without interfering with the short-range communication channel [1, 21]. The communication among actors in different actor sets uses sensor nodes to relay its data to the target actor. Source actor nodes can switch to a sensor communication channel with a longrange transmission to reduce the number of hops and number of intermediate sensor nodes required to act as a bridge between actor nodes $[3,8]$, but the communicate rate is $v_{\mathrm{s}}$ in this time.

(3) All sensor nodes do not move after being deployed $[1,21]$. On detecting an event, a sensor node will generate messages and send those messages to one of the actors $[1,21]$. Actor makes decision after the actor receives the information of merging other actors, so it needs to exchange event information among actors. We consider the probability of the communication among any two actors is equal. Therefore, the communication among actors in a set adopts AAc style, and the communication among actors in different actor sets uses ASAc style.

\subsection{Energy consumption model and related definition}

According to the typical energy consumption model [3, 7 12], energy consumption for transmitting (see Eq. (1)) and energy consumption for receiving are represented by Eq. (2).

$$
\begin{aligned}
& \begin{cases}E_{\text {member }}=l E_{\text {elec }}+l \varepsilon_{f s} d^{2} & \text { if } \quad d<d_{0} \\
E_{\text {member }}=l E_{\text {elec }}+l \varepsilon_{\mathrm{amp}} d^{4} & \text { if } \quad d>d_{0}\end{cases} \\
& E_{R}(l)=l E_{\text {elec }}
\end{aligned}
$$

$E_{\text {elec }}$ represents transmitting circuit loss. If the transmission distance is less than the threshold $d_{0}$, the free space ( $d^{2}$ power loss) are used in the model. When the transmission distance is greater than or equal to the threshold, the multi-path fading ( $d^{4}$ power loss) channel models are used in the model. $\varepsilon_{\mathrm{fs}}$ and $\varepsilon_{\mathrm{amp}}$ are respectively the energy required by power amplification in the two models. The energy consumption for receiving $l$ is the number of bit of packet. The above parameter settings are shown in Table 1, as adopted from $[5-7,9,10]$.
Table 1 Network parameters

\begin{tabular}{ll}
\hline Parameter & Value \\
\hline Threshold distance $\left(d_{0}\right)(\mathrm{m})$ & 87 \\
Sensing range $r_{s}(\mathrm{~m})$ & 15 \\
$E_{\text {elec }}(\mathrm{nJ} / \mathrm{bit})$ & 50 \\
$e_{\mathrm{fs}}\left(\mathrm{pJ} / \mathrm{bit} / \mathrm{m}^{2}\right)$ & 10 \\
$e_{\text {amp }}\left(\mathrm{pJ} / \mathrm{bit} / \mathrm{m}^{4}\right)$ & 0.0013 \\
Initial energy $(\mathrm{J})$ & 0.5 \\
\hline
\end{tabular}

\subsection{Problem statement}

The main goal of this paper is to alleviate this problem that ASAc cannot satisfy the practical application, because the communication ability of sensor nodes in the actor-sensor-actor is less than the actor-actor. This results in the small communication capacity of the sensor nodes in the middle area, which limits the application of the network. A high-throughput disjoint multi-path (HTDM) routing scheme is proposed to bridge the gap of ASAc so as to meet the requirements of application. HTDM routing scheme needs to achieve the following goals:

(1) Bridge the gap of communication bottleneck. In this paper, the main problem needs to be solved is to alleviate the communication capabilities difference between actors and sensor nodes. The communication capabilities among actors are $v_{\mathrm{a}}$, and the actor-sensor-actor communication (ASAc) capabilities is $v_{\text {asa }}$. Let $\varsigma=v_{\text {asa }} / v_{\text {a }}$ represent the mismatch degree between $v_{\text {asa }}$ and $v_{\text {a }}$. Obviously when $v_{\text {asa }}=v_{\mathrm{a}}, \varsigma$ is 1 . It illustrates that communication capabilities of actor-sensor-actor is equal to the actor's maximum value. And the worst case is $v_{\text {asa }}=v_{\mathrm{s}}$, at this point, the communication capabilities of actor-sensor-actor is the same to communication capabilities among sensor nodes. Therefore, routing strategy of this paper is to make $\varsigma$ maximum, namely:

$\min (\varsigma)=\min \left(v_{\mathrm{asa}} / v_{\mathrm{a}}\right)$

(2) Maximize the network lifetime. The fundamental goal of application is to make the network lifetime maximum. The network lifetime can be defined as the time of the node that dies first. Because after the first node dies, it is likely that they will seriously affect the network connectivity and coverage, which lead to network cannot fully play their role. Therefore, the same as Ref. [24], the network 
lifetime can be defined as the time of the node that dies first. Set $E_{\mathrm{i}}$ is the energy consumption of node $i$, so the network lifetime maximization can be expressed:

$$
\max (T)=\min \max _{0<i \leq n}\left(E_{i}\right)
$$

(3) Data collection with low delay. For real-time transmission, data packets should be transmitted to sink as fast as possible. The concept of delay is different for different nodes. Specifically, due to the same communication ratio among sensor nodes, the communication delay is affected by the length of the routing path, the longer the length of route path is, the longer the delay is, otherwise, the delay is small $[19,20]$. In this paper, the delay is defined as the requirement time for transmitting unit data when actor sets communicate with other actor sets. Obviously, the longer the $\varsigma$ is in actor-actor communication, the smaller the corresponding delay is. Let the time $t_{1}$ represent the requirement time for transmitting unit data among actors, that is $t_{1}=1 / v_{\text {asa. }}$. Let the time $t_{2}$ represent the time for establishing routing path among actors. Delay is defined as $D=t_{1}+t_{2}$. Optimization goal is to make the delay minimum, namely $\min (D)=\min \left(t_{1}+t_{2}\right)$. To sum up, the optimization goal of this paper can be obtained:

$$
\left\{\begin{array}{l}
\max (T)=\min \max _{0<i \leq n}\left(E_{\mathrm{i}}\right) \\
\max (\varsigma)=\max \left(v_{\mathrm{asa}} / v_{\mathrm{a}}\right) \\
\min (D)=\min \left(t_{1}+t_{2}\right)
\end{array}\right.
$$

\section{The high-throughput disjoint multi-path scheme} If communicating actor sets belong to different partitions, the long-range actor channel cannot be utilized. It is essential that intermediate sensor nodes are involved in establishing the communication between source nodes and destination nodes [1, 21]. Because the communication capability of actor is greater than sensor node, the multi-path routing method is adopted to fill the gap in this paper. The HTDM routing scheme mainly contains the following two stages: (1) initial stage. At this stage, each node obtains the shortest hop to each actor set through the hop count diffusion protocol [12]. (2) The stage of multi-path routing. When nodes in different actor sets need to communicate with other nodes, they can communicate through creating multi-path routing between source nodes and destination nodes.

\subsection{Building the hop for actors and sensors}

The main purposes of HTDM routing scheme in the first stage are

(1)The formation of actor set and routing among actors in the same actor set.

At this stage, each actor can broadcast their routing information with the communication radius $r_{\mathrm{a}}$, the format of broadcast packets are

$$
\left\{I D_{a},\left(I D_{1}, I D_{1, n}, \text { hops }\right),\left(I D_{2}, I D_{2, n}, \text { hops }\right), \ldots,\left(I D_{k}, I D_{k, n}, \text { hops }\right)\right\}
$$

Broadcast packets are composed of the actor's own ID and multiple three tuples as Eq. (6). The composition of each three tuples (ID, next ID, and hop number) denote the target actor's ID, the ID of the next actor, and the hop count from the node to the target actor, respectively. Element $I D_{\mathrm{a}}$ represents the ID of actor which generate broadcast packet. The information can only contain their own ID number in the source node, in which the content of packet is $M_{\mathrm{a}}=\left\{I D_{a},\left(I D_{a}, I D_{a}, 0\right)\right\}$. After the other actors in the communication range receive broadcast packet, it will compare the information in broadcast package with his message. If the actor found the information, which the actor can reach to another new actor or have a shorter route to other actor, it will update its own message. The updated principle is (1) if there is a new target actor's ID in broadcast package, a new three tuples are created, in which the first item is the ID of new target actor; the second item is the ID of actor which generates the broadcast message, and the third changed to hops +1 , that is to say the actor which produce broadcast packet can reach to a new actor by hops +1 hops. Then, the actor adds the new tuple to his message. (2) If the actor found that the number of tuples is the same in the broadcast packet and the target actor is the same, but hops +1 is smaller than the current hop count hops, then the three tuples can be updated. The method for updating is to change the second item of the three tuples to the ID of broadcast source actor, and to change the content of the three items to hops of the third item with the tuple in received broadcast packets add 1 . As long as there is update for broadcast packet in an actor, actor will broadcast packet after waiting for a suitable time slots. After a period of time, until the broadcast information of each actor no longer need to be updated, the routing path is formed between actor set and actor. Finally, as every actor knows the each actor's ID in all the actor sets, the function $f$ is used to get actor set ID whose input is all ID in an actor set. This process is described by algorithm 1 . 
Algorithm 1: Form the actor set and obtain the hop count to any actor and the next-hop node in the same actor set Input: WSANs

Output: the actor in an actor set has the same actor set ID, the actor save the minimum hop count and the next hop node to other actor 1 For each actor $a \in A_{\mathrm{a}}$ do

2 Set actor $a^{\prime} s$ broadcast message $M_{a}=\left\{I D_{a},\left(I D_{a}, I D_{a}, 0\right)\right\} \quad / /$ Message is their own ID number, the ID of next hop nod //and hop count, expressed by (ID, next ID, hop number).

3 Set actor $a^{\prime} s$ broadcast time $t_{a} \in\left\{0, t_{b}\right\} \quad / / t_{b}$ is the maximum broadcast time slot

4 End For

5 For each actor $a \in A_{\mathrm{a}}$ which is in broadcast time slot and can broadcast without collision Do

$\left.6\right|_{7 \text { End For }}$ broadcast its message

\section{For each actor $a \in A_{\mathrm{a}}$ that received a message $M_{i}$ from actor $i$ Do}

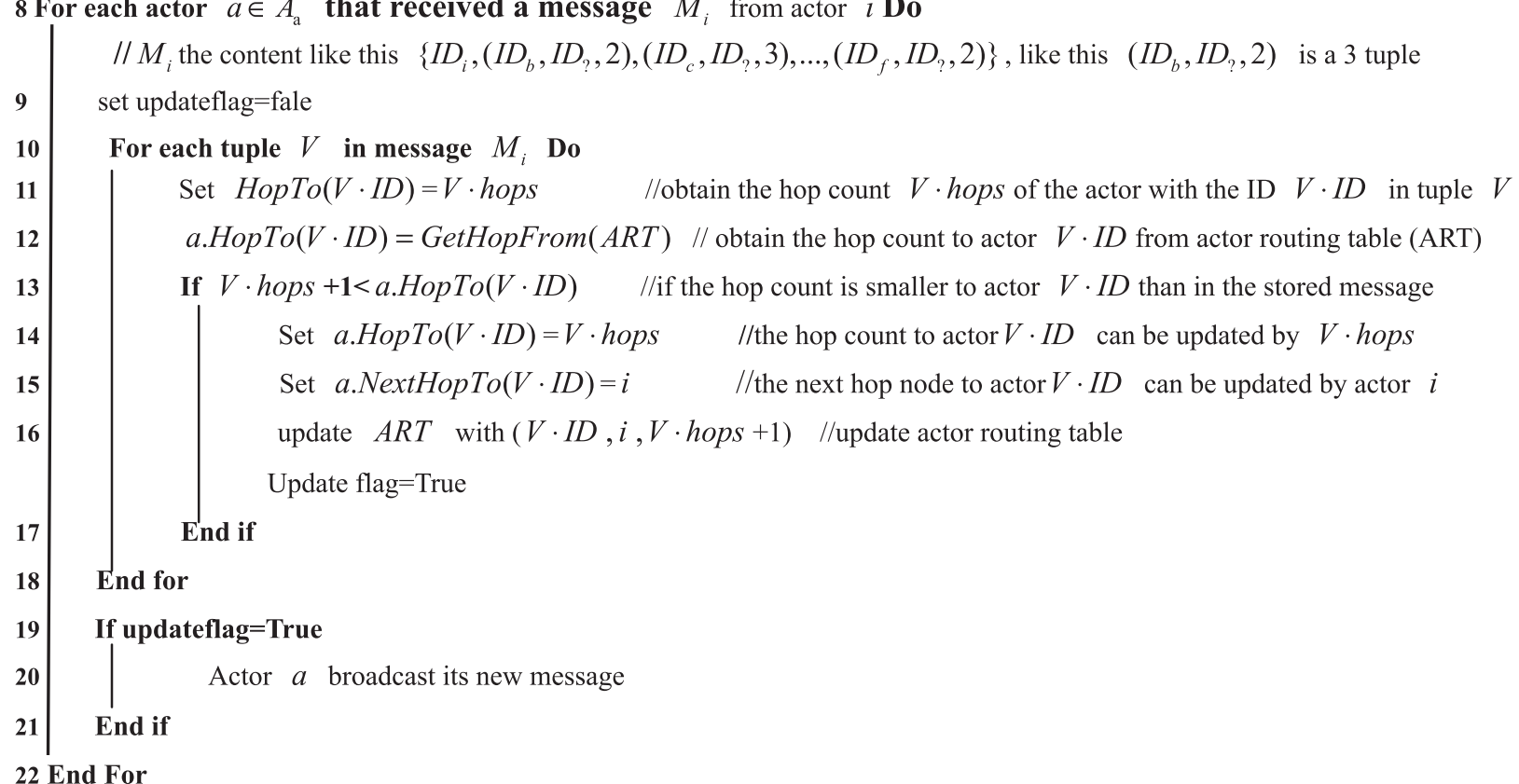

23 For each actor $a \in A_{\text {a }}$ Do

$24 \quad$ Set its actor set ID is $f($ all id in $A R T) \quad$ //actor set ID are mapped by the function $f$ 25 End For

(2) The routing from sensor nodes to each actor and actor set are formed.

Every actor node in each actor sets switch to a sensor communication channel with long-range transmission to broadcast themselves information. The sensor nodes in the communication range of actor can receive broadcast information of actors. Thus, actors can send data to those nodes directly, so these nodes mark themselves as actor direct transmission area (ADTA), and set the hop count from themselves to this actor set is 0 ; the distance to all the actors in other actor set is $\infty$. After the information are broadcasted, the hop count from all sensor node in ADTA to their actor set is 0 , the distance to the actor in other actor sets is infinity. Sensor nodes $s_{1}, s_{7}$ in Fig. $1 \mathrm{~b}$ belong to ADTA of actor set 2 (AS2); sensor nodes $s_{5}, s_{6}, s_{11}, s_{12}$ belong to ADTA of actor set 4 (AS4).

Then, each actor broadcasts message (packet) with $\left\{I D_{\mathrm{a}}, I D_{\mathrm{as}}, 0\right\}$. The 0 of the message means that the hop count to himself and his actor set is 0. All the sensor nodes look for his sensor routing table $\left(\mathrm{SRT}_{s}\right)$ when receiving this message. The sensor node compare the hop count to the located actor set with the hop count in broadcast packet. If the hop count in the node is bigger than in the broadcast, the corresponding table is updated. If a sensor node updates 


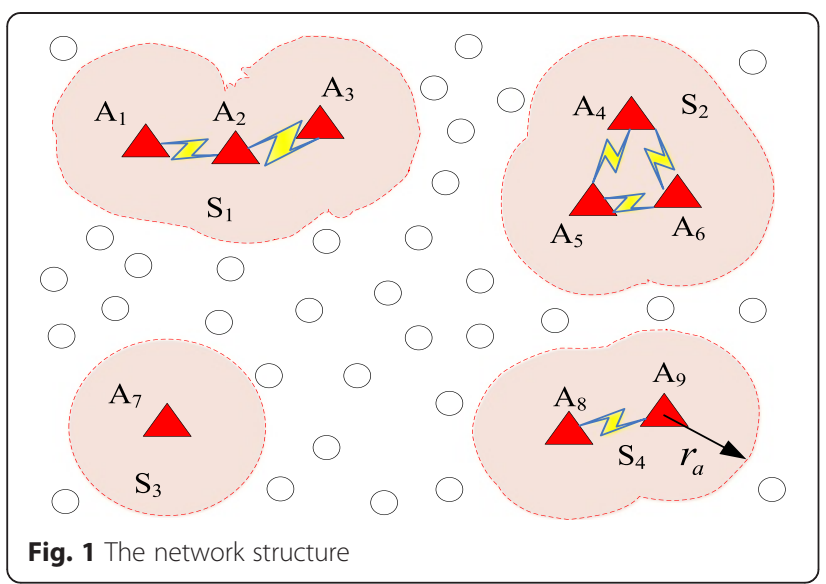

its own table, after a period of time, the sensor node broadcast his routing tables. After all, its neighbor nodes receive the broadcast packet, the nodes see whether there is a need to update their routing table. This process repeats until each sensor node determines the minimum hop count and next hop node to all actors and actor sets which is described by algorithm 2 .

\subsection{Building multi-path between source-destination actor set pair}

(1)If all the actors are located in the same actor set, its routing method is as follows:

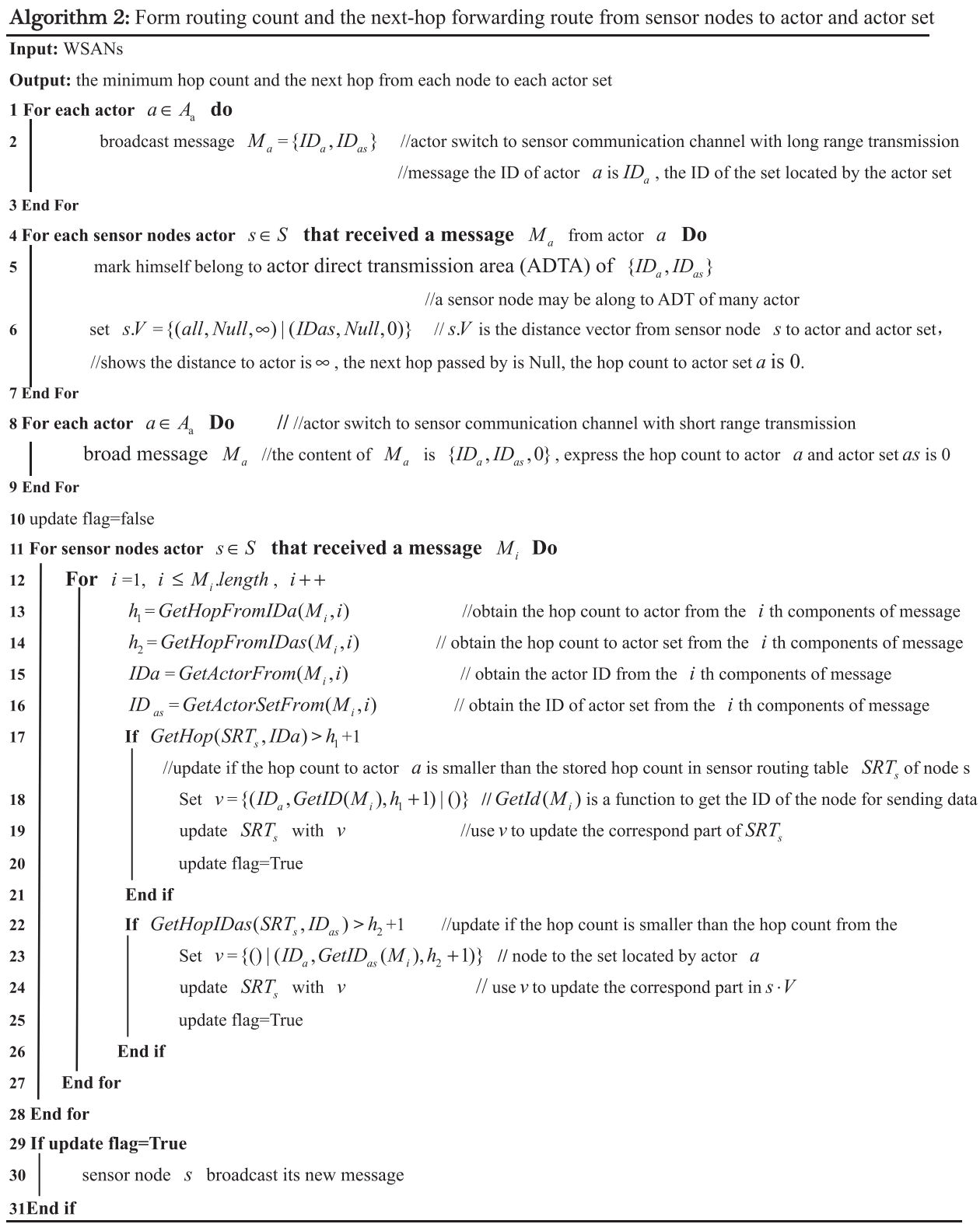


The main idea for creating multi-path routing is: for the communication among actors in the same actor set, algorithm 1 gives the minimum hop count from one actor to each actor in the same actor set and the next node according to the principle of shortest path, so the actors in the same actor set can communicate with each other according to the shortest routing method. The algorithm is shown in algorithm 3 .

(2) The communication among actors in different actor set, its routing approach is as follows:

The fact that the difference of communication ability is huge between actors and sensor nodes, thus, as long as the data of actor $a_{1}$ are sent to any actor in the actor set located by actor $a_{2}$, it does not affect communication throughput. There is no bottleneck of actor-actor communication. For the sender, due to the communication capacity among actors are stronger than sensor node, when the sender actor wants to communicate with other actor, the actors are adopted as the relay nodes unless when the communication cannot be bypass by sensor node. Therefore, in the HTDM, although the actor communicate with the other actor in different actor sets, our main goal is to send the data in any actor located by sender actor to any actor node in the actor located by receiver actor. Because, it can be communicated using algorithm 3 when the actor inside of the sender actor set and the receiver actor set. So, the algorithm 4 can only consider how to send the data in actor set located by sender actor to the actor set located by receiver actor.

Due to the ability of the actor is bigger, and it has GPS positioning information, etc. So, all the actors in the actor set know the hop count from each sensor node in actor direct transmission area (ADTA) to the actor set located by receiver actor. In HTDM scheme, the approach for creating first routing path is as follows: (1) The sponsor actor $a_{1}$ select sensor node $s$ in the range of the ADTA and the minimum hop count to actor set located by receiver actor as the starting node of the first routing path. If the sender actor $a_{1}$ cannot communicate with sensor node directly, then the actor $a_{1}$ routes the data packet to the actor which can communicate directly with the sensor node $s$. Then the data packet can be sent to the sensor node $s$. The reason is that the communication capacity among actors is much higher than sensor node, and the communication that passes by the actor is the most fast and save energy style. (2) The sensor node $s$ routes the data packet to the area of ADTA located by target actor $a_{2}$ using the shortest routing algorithm. The sensor nodes in ADTA route the data packets to the actor nearest to destination actor using the shortest routing algorithm. This actor routes the data packets to target $a_{2}$ using routing algorithm 3 . (3) In the process of creating a routing path, if the sensor nodes in the range of one hop of routing path eavesdropping the information about creating routing path, then record the ID information of the routing path. This means that this node is in the range of communication interference of the routing path.

Then more routing paths base on the created routing path can be created. The method for creating other routing paths is are follows: (1) sensor node with minimum hop to target actor set, which is not in the range of interference of the created routing path, such as, node $b$, is selected as the next hop node by sender actor $a_{1}$. If sensor node $b$ cannot communicate with sender actor $a_{1}$, actor $a_{1}$ routes data packets to sensor node $b$ through other actors

Algorithm 3: The routing algorithm of actor in the same actor set

Input: the actor need communicate with other actor in an actor set, The sender is actor $a_{1}$, the receiver is actor $a_{2}$

Output: the routing path of actor $a_{1}$ and actor $a_{2}$

1 Let $I D_{1}=$ GetActorID $\left(a_{1}\right), I D_{2}=$ GetActorID $\left(a_{2}\right) \quad$ //obtain the ID of the node according to the name of the node

2 Let $N_{\text {next }}=$ GetNextActor $I D\left(I D_{1}, I D_{2}\right) \quad$ //look for the next hop node to node $I D_{2}$ from vector table $V$ from node $I D_{1}$

//to other actor

3Send packet to node $N_{\text {next }}$

$/ /$ node $I D_{1}$ send data packets to node $N_{\text {next }}$

4 While $N_{\text {next }}$ is not $I D_{2}$

$5 \quad N_{\text {next }}=$ GetNextActorID $\left(N_{\text {next }}, I D_{2}\right) \quad$ //obtain the next hop node from actor $N_{\text {next }}$ to $I D_{2}$

6 Send packet to $N_{\text {next }}$ 
Algorithm 4: The multi-path routing algorithm among different actor sets Input: the communication between actor in different actor sets, sender is actor $a_{1}$, the receiver is actor $a_{2}$, create $k$ multi-paths Output: the routing path of actor $a_{1}$ and actor $a_{2}$

1 Let $I D_{a s 1}=\operatorname{GetActorIDas}\left(a_{1}\right), I D_{a s 2}=\operatorname{GetActorIDas}\left(a_{2}\right) / /$ obtain the ID of actor set located by node according to his name

2 Let $s=$ LeastHopNode $\left(I D_{a s 2}\right) \quad / /$ sensor node $s$, which is the minimum hops counts to reach the actor //set $I D_{a s 2}$ within actor $a_{1}$ ADTA

3 Let $a_{s}$ is the actor that can directly send data to $s$

4 Send packet to actor $a_{s}$ use algorithm 3

5 Create $I D_{p}$ for this multi- path

// Create unique route ID for this multi path

$6 \quad N_{\text {next }}=$ GetNextSencorToAS $\left(N_{\text {nexxt }}, I D_{a s 2}\right) \quad$ //obtain the next hop node to actor set $I D_{a s 2}$ through looking for table $S R T_{s}$

7 While $N_{\text {next }}$ is not in ADTA of receiver

$8 \quad N_{n e x t}=$ GetNextSencorToAS $\left(N_{\text {nexxt }}, I D_{a s 2}\right) \quad$ //obtain the next hop node from actor $N_{\text {next }}$ to $I D_{\text {as } 2}$

$9 \quad$ Send packet to $N_{\text {next }}$

10 End While //reach the ADTA area of actor set located by actor $a_{2}$

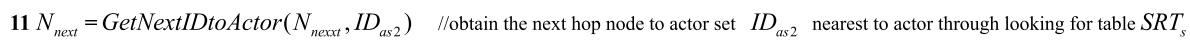
12 While $N_{\text {next }}$ is not actor $I D_{a 2}$

$13 \quad N_{\text {next }}=$ GetNextIDtoActor $\left(N_{\text {next }}, I D_{a s 2}\right) \quad / /$ choose the sensor node nearest to actor as next hop node

14 Send packet to $N_{\text {next }}$

15 End While //reach an actor in actor set located by actor $a_{2}$

16 If $N_{\text {next }}$ is not actor $a_{2}$

17 Send packet to actor $a_{2}$ use algorithm 3 //send data packet to target actor

18 End if

19 For each sensor node that can eavesdropping the path creating //those nodes are in the impact range

20 Record the path information

21 End for

$22 i=\mathbf{1}, j=0, \zeta=$ Null

23 While $i<k$ and $j<2 \quad$ //create other $k-1$ routing paths

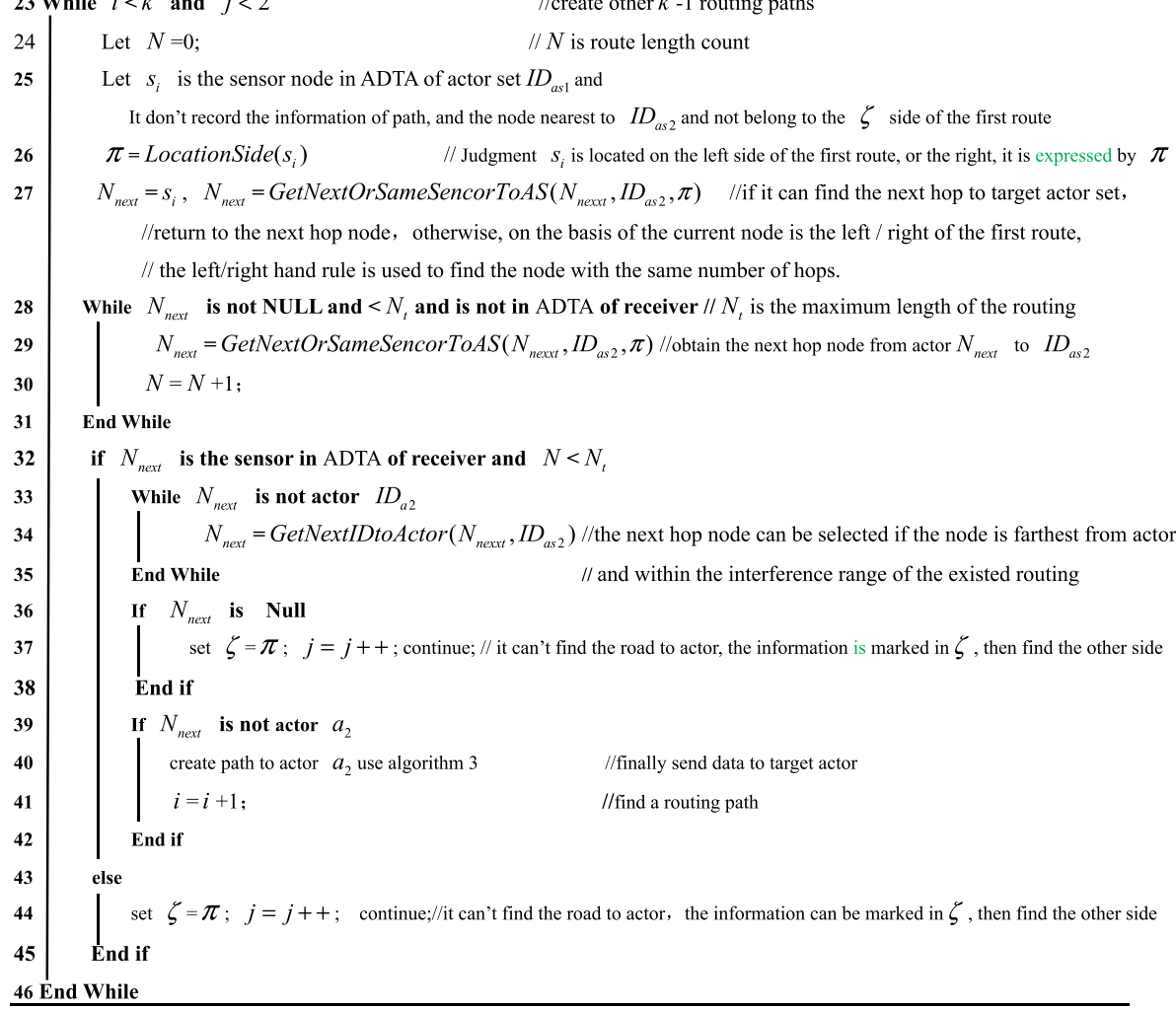


using algorithm 3. (2) Creating a new routing path from sensor node $b$. Sensor node can be selected as the next hop node by sensor node $b$ using the same method. If a node cannot be found by sensor node $b$, but sensor node $b$ is the left side of the first routing path, sensor node, which have same hop count with him and in the left side of sensor node $b$ can be selected as the next hop node by sensor node $b$ (the equivalent of the left hand rule around an empty routing algorithm). (3) After the data packets are routed to the area located by target actor $a_{2}$, sensor nodes in ADTA route data packets to the actor nearest to destination actor according to the shortest routing algorithm. Then those data packets can be routed to target actor $a_{2}$ using algorithm 3. (4) Repeat the above (1)-(3) to create a new routing path; (5) in the process of creating routing path, it marks the influence range of routing. If the node eavesdropping routing message of creating routing path, it records the ID information of the routing path, this said it is in the range of communication interference of this routing path. Algorithm 4 shows the algorithm for creating multi-path.

Figure 2 shows the process of establishing multipath routing, such as Fig. 2a, considering that actor $A_{6}$ has data needs to be sent to actor $A_{8}$. Each sensor node can get the hop count to any actor using algorithm 1 and algorithm 2. The digital of the nodes in Fig. 2 can express the minimum hop count to the actor in actor set $4\left(A S_{4}\right)$. Because the hop count is minimum from sensor node $s_{1}$ to the actor in $A S_{4}, s_{1}$ is chosen to the first sensor node of the first routing path. The node, which distance from $A S_{4}$ is smaller than node $s_{1}$, is selected as the next hop node by node $s_{1}$. So the first routing path is established as Fig. 2a firstly, data packets of $A_{6}$ are sent to $A_{5}$. Second, those data packets are sent to $A_{9}$ along the first routing path. Finally, the data are sent to target

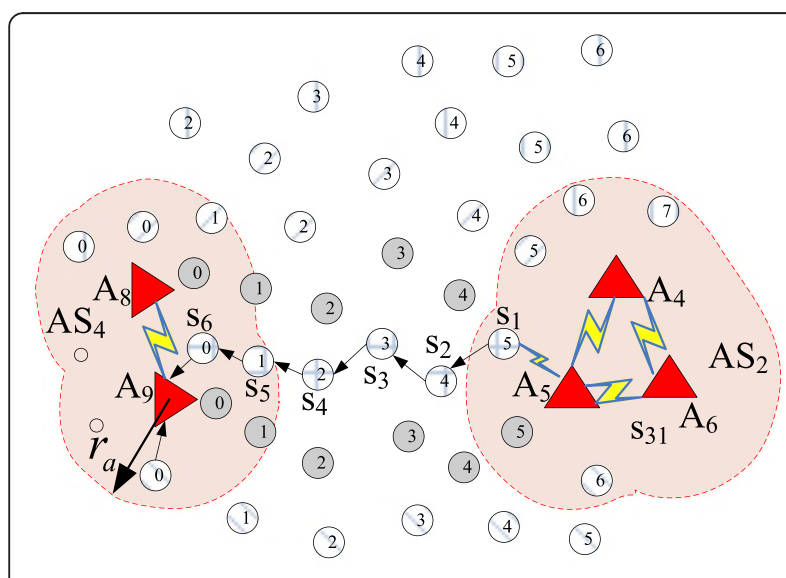

(a) Establish the first route path

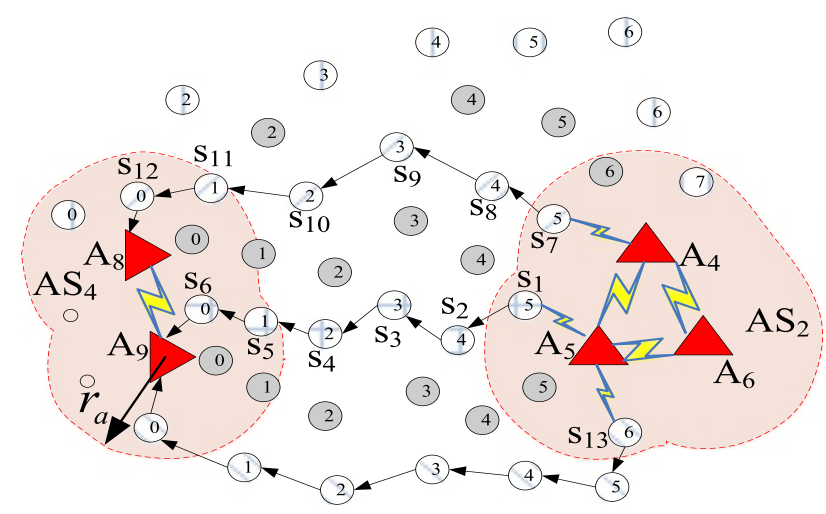

(c) Establish the third route path

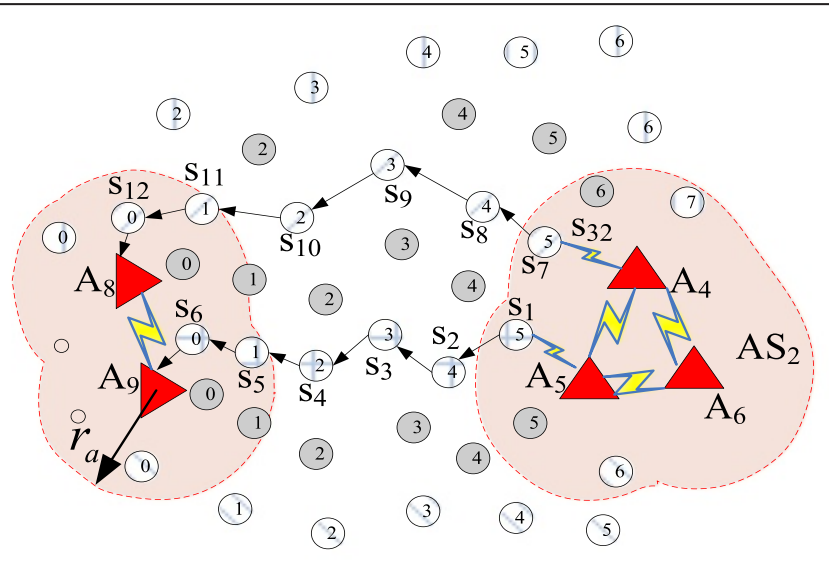

(b) Establish the second route path

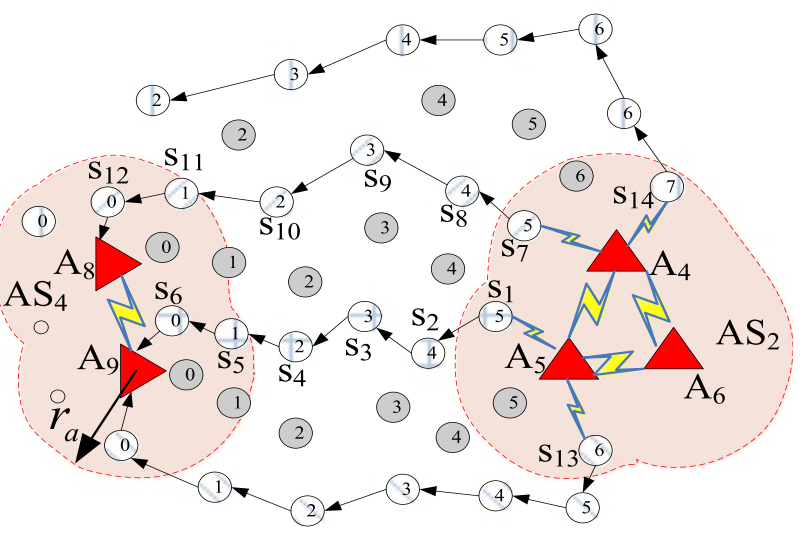

(d) The forth route path can not be established

Fig. 2 Illustration of the HTDM routing scheme. a Establish the first route path. b Establish the second route path. $\mathbf{c}$ Establish the third route path. d The forth route path cannot be established 
actor $A_{8}$. At the same time, the nodes in the range of interference of first routing path are marked as grey. The grey nodes are not selected in the latter of creating routing path.

Second, it turn to create the second routing path, at this point, the first routing path and interference nodes in the first routing path are not selected as the intermediate node. So, the remaining node nearest to $A S_{4}$ is $s_{7}$, node $s_{7}$ can be selected as the first node in the second routing path. Then, node $s_{7}$ creates the routing path as Fig. 2b based on the shortest routing path method. In this way, the routing of data transmission is firstly, data packets of $A_{6}$ are sent to node $A_{4}$. Second, those data packets are send to node $A_{8}$ along the second routing path. According to algorithm 4, sensor node $s_{13}$ is the best node as the first sensor node of creating the third routing path, so, you can create the third routing path, such as Fig. 2c. But the forth routing path cannot be created, such as Fig. 2d.

\subsection{The load balancing multi-path routing}

Multi-path routing is proposed to bridge the gap among the actor-sensor-actor communication (ASAc). But the proposed routing policy is only able to build multiple routing paths, but not consider the balanced problem of the energy consumption, which can damage the network lifetime. A practical example to explain the question in the following. For the network diagram in Fig. 1, according to the above algorithm, the routing of the network communication among four actor sets are routed along the shortest routing path. If the communication probability among each actor sets is equal, due to the central region of the network is the intersection area of $A S_{1}$ and $A S_{4}, A S_{2}$ and $A S_{3}$, so the energy consumption in the intersection area is higher than other areas.

Figure 4 shows the result of the experiment. For the network diagram as Fig. 3, when $A S_{4}$ communicates with $A S_{1}$, and $A S_{3}$ communicates with $A S_{2}$, the energy consumption in the intersection area is much higher than other regions (see from Fig. 4), which affect the network lifetime. In fact, when $A S_{4}$ communicates with $A S_{1}$, the length of the routing path is not always the shortest, it also can choose the other path to route data to balance the energy consumption. For example, when $A S_{4}$ sends data to $A S_{1}$, it also can send data to $A S_{2}$, then send data to $A S_{1}$. This can balance the network energy consumption.

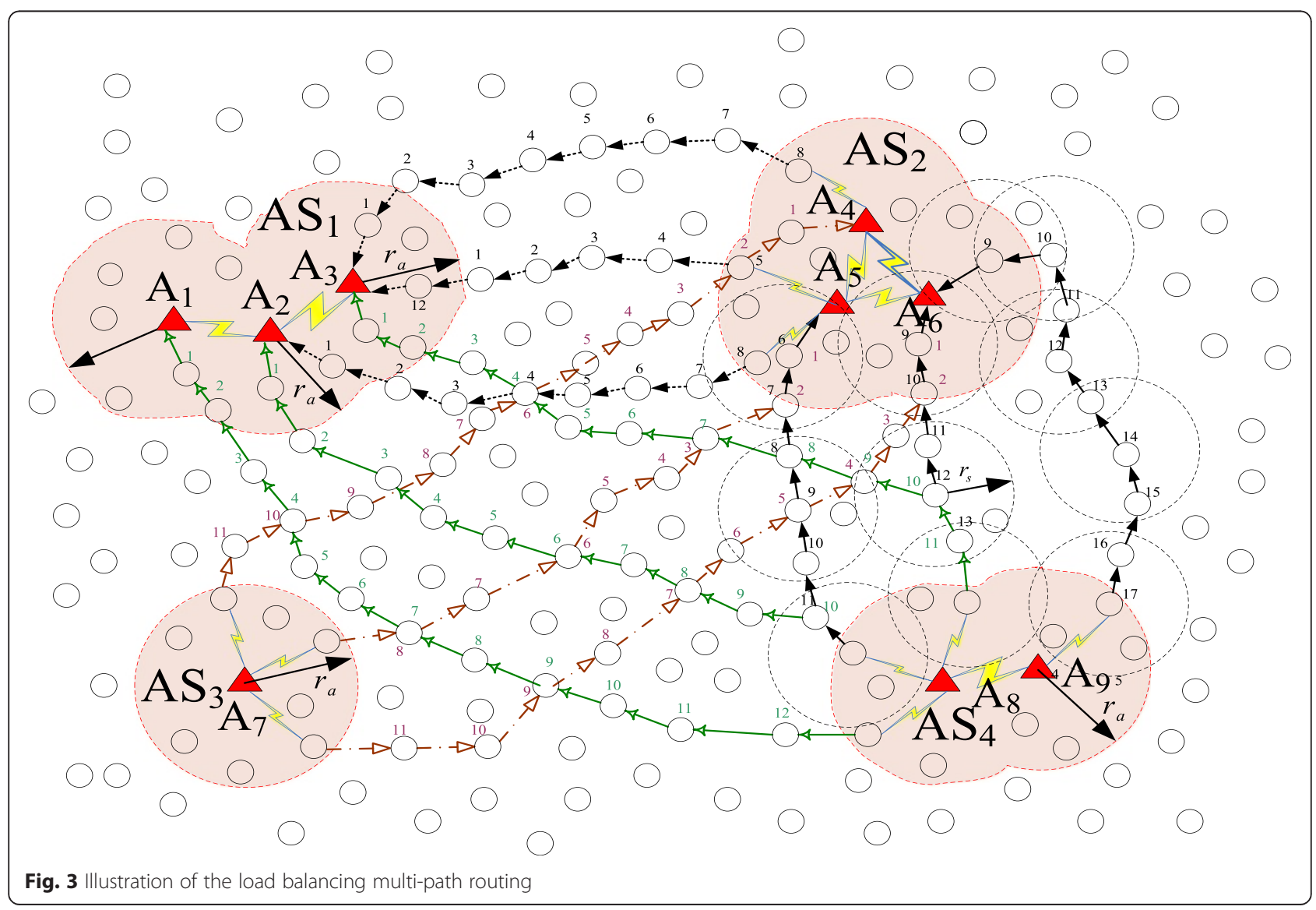




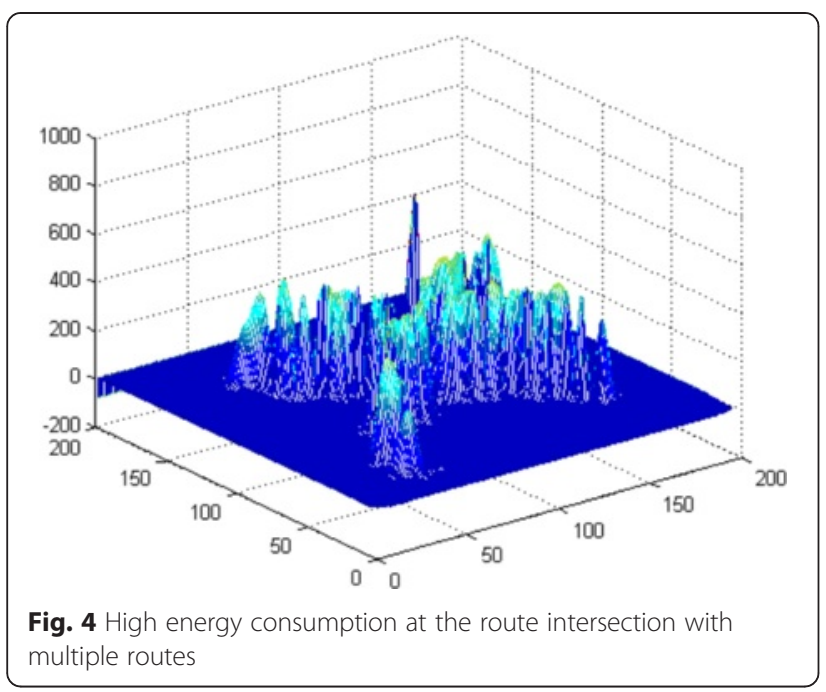

Therefore, the proposed multipath routing algorithm can be improved in this section; the improved algorithm can increase the ability of balancing the energy consumption, the improved algorithm called enhanced high-throughput disjoint multi-path (EHTDM) routing scheme. The difference between EHTDM and HTDM scheme are (1) when actor create routing path, the next hop node can be selected according to the principle with minimum hop counts and maximum residual energy. (2) In the previous schemes, the node can be selected as the next-hop node according to shortest path, which can cause that the energy consumption in these routing areas are much higher than other areas. A scheme is proposed to balance the energy consumption of the network; this method is that if the number of creating multiple routing path is $k$, when data packet needs to be sent to sink, $k$ routing path with lowest energy consumption can be selected by the node to transmit data. Because looking for much paths need to consume more energy, it cannot be used regularly. Thus, the way of looking for the much possible paths is to be executed at set intervals in EHTDM scheme.

An enhanced high-throughput disjoint multi-path routing is illustrated in Fig. 5. For example, four AS regions are arranged in a row in Fig. 5. Considering the probability of communication among actors is equal, because there are three actors in $A S_{1}$; three actors in $A S_{2}$, and one actor in $A S_{3}$, and two actors in $A S_{4}$. Therefore, the probability of routing between $A S_{4}$ and $A S_{2}$ is $2 \quad 3=6$, the probability of routing between $A S_{4}$ and $A S_{3}$ is $2 \quad 1=2$, the probability of routing between $A S_{4}$ and $A S_{1}$ is $2 \quad 3=6$, the probability of routing between $A S_{2}$ and $A S_{3}$ is $3 \quad 1=3$, the probability of routing between $A S_{2}$ and $A S_{1}$ is $33=9$, the probability of routing between $A S_{3}$ and $A S_{1}$ is $1 \quad 3=3$. So comprehensively, the probability of routing assumed by the nodes between $A S_{3}$ and $A S_{1}$ is 14 ; the probability of routing assumed by the nodes between $A S_{2}$ and $A S_{3}$ is $8+12=20$, the probability of routing assumed by the nodes between $A S_{2}$ and $A S_{3}$ is $3+9+6=18$. Therefore, the energy consumption in hotspots area of red area in Fig. 5 is the

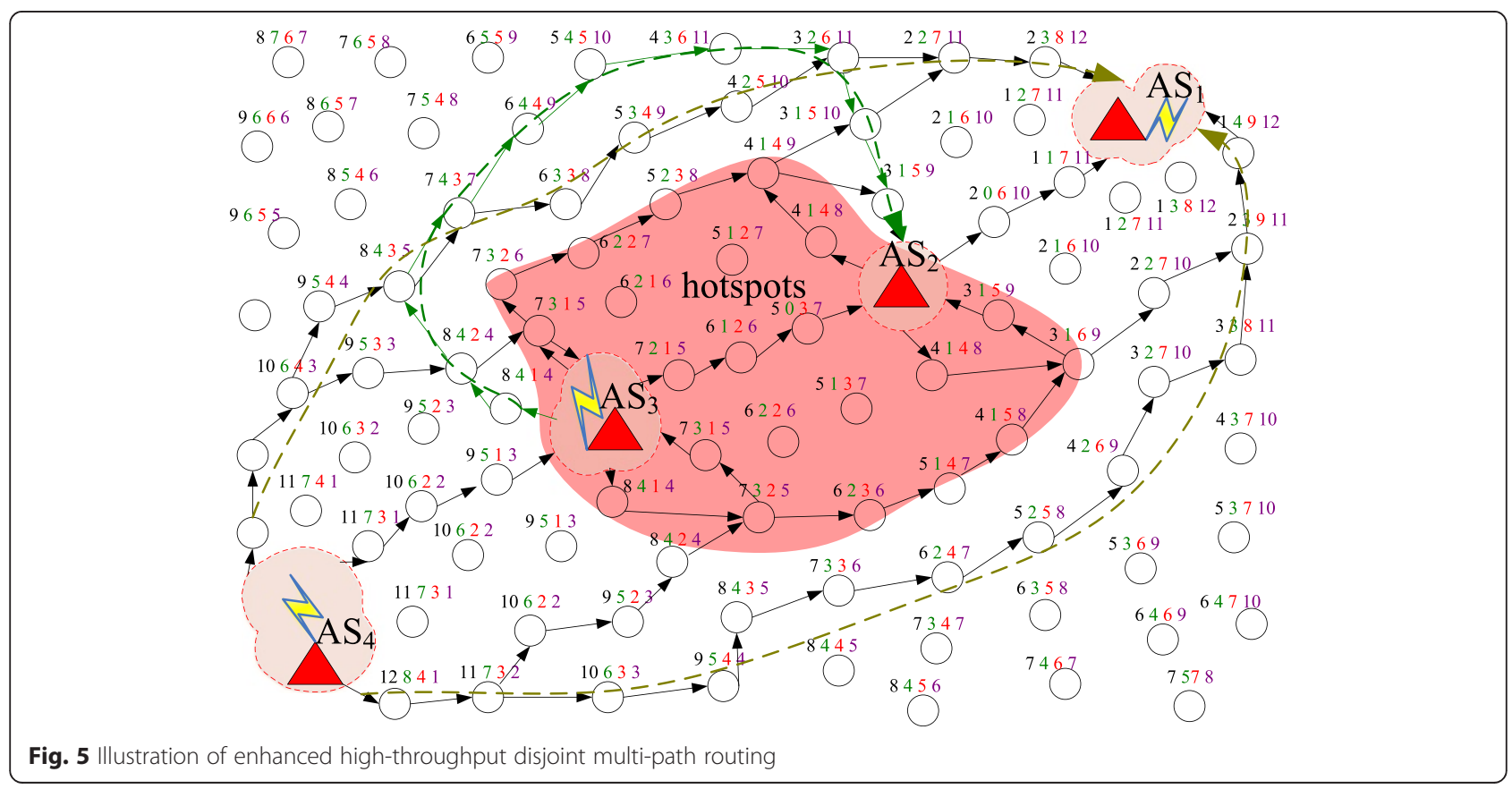


Algorithm 5: Enhanced high throughput disjoint multi-path routing algorithm

Input: the communication between actor in different actor sets, spender is actor $a_{1}$, the receiver is actor $a_{2}$, create $k$ multi-paths

Output: choose $k$ routing paths with minimum energy consumption from actor $a_{1}$ to actor $a_{2}$

1 use algorithm 4 get more routing path as much as possible from actor $a_{1}$ to actor $a_{2}$ with the condition of the following

2

condition 1: If there are more than one candidate nodes for the next hop routing, it selects the node with maximum remaining energy

as the next hop.

3 condition 2: the nodes with the maximum energy consumption in each route are recorded.

4 output the $k$ routing paths with minimum energy consumption in the routing

highest. If the system adopts HTDM routing scheme, the nodes in hotspots area will die in advance because of the high energy consumption. But in EHTDM routing scheme, when the system is running for a period of time, EHTDM scheme searches routing path as much as possible, and then select the routing path with the lowest energy consumption to transmit data. Thus, for the WSANs of Fig. 5, when $A S_{4}$ communicates with $A S_{1}$, it will choose the routing path without passing the hotspots area to transmit data, such as the brown routing path of Fig. 5 . When $A S_{2}$ communicates with $A S_{3}$, in order to bypass hotspots area, EHDM scheme will choose green routing path in Fig. 5 to communication, which balances the energy consumption of the network and prolongs the network lifetime. Here are EHTDM routing algorithms.

\section{Experimental evaluation}

OMNET++ is used for experimental verification which has been widely recognized by academics [51]. The network topology adopted in this paper can be seen from Figs. 1 and 5. In the following section, the network scenario in Fig. 1 is denoted as experimental scenario (1), and the network scenario in Fig. 5 is denoted as experimental scenario (2). To the best of our knowledge, there are no similar researches to bridge the gap among the actor-sensor-actor communication (ASAc) using the multi-path routing, thus, this experiment mainly verify the following questions: (1) The proposed HTDM and EHTDM routing scheme is feasible. It is mainly illustrated through the experiment of section 5.1. (2) The throughput situation of HTDM and EHTDM routing scheme, that is, the comparison of the number of transmitting data in a fixed time between the proposed scheme and other multi-path routing scheme. (3) The comparison of data transmission delay between HTDM and EHTDM routing scheme. That is, the comparison of the requirement time when transmits a certain number of data between the proposed scheme and other multi-path routing scheme. (4) The comparison of network

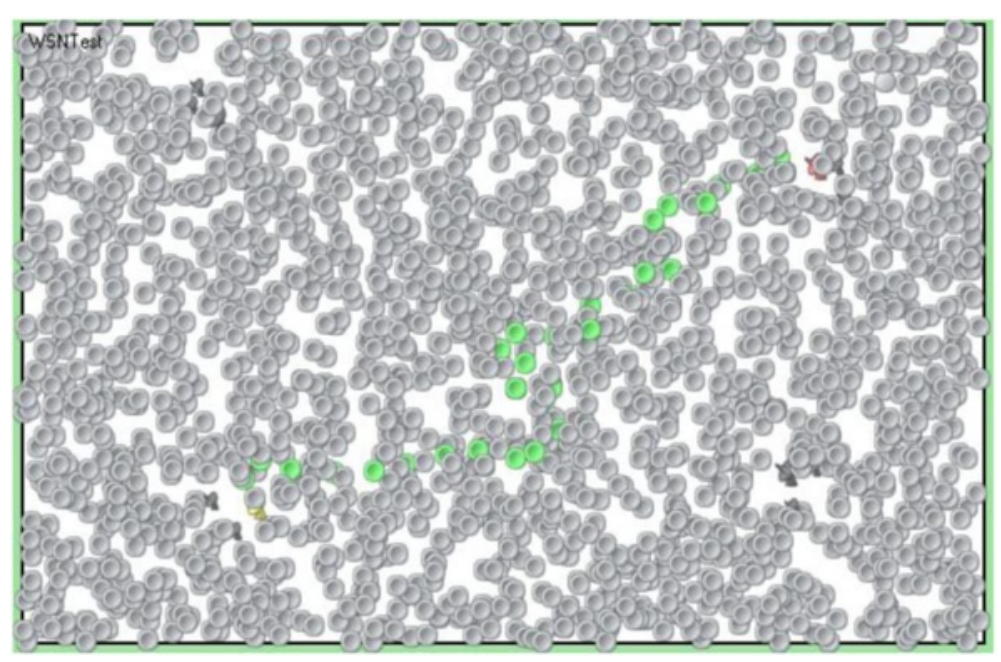

Fig. 6 Single-path routing experiment (scene 1) 


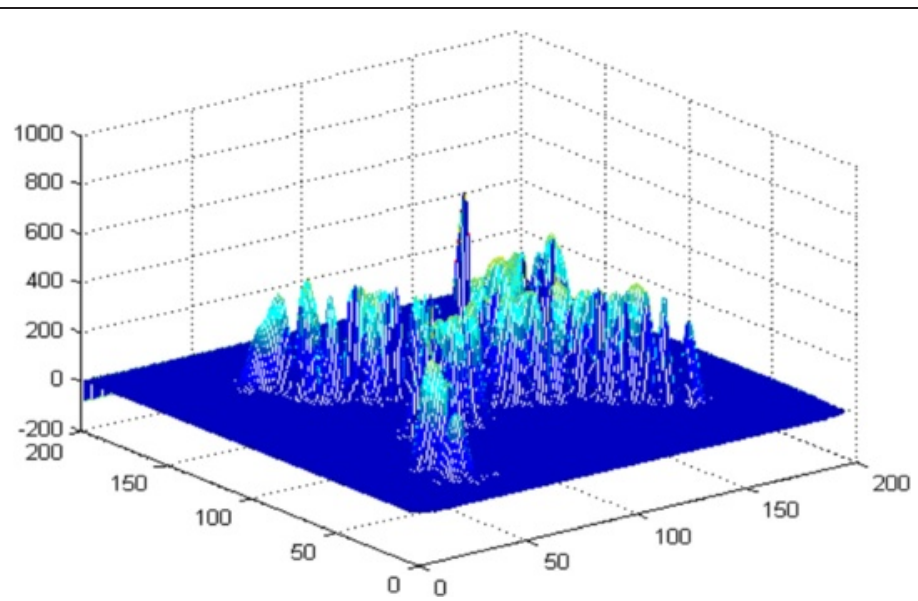

Fig. 7 Energy consumption of the network under the single path routing strategy (scene 1)

lifetime between HTDM and EHTDM routing scheme, it can demonstrate that the EHTDM routing scheme can improve the network lifetime due to balance the energy consumption.

\subsection{The energy consumption and running of HTDM routing scheme}

The experimental results in the paper are mainly to verify the running situation of HTDM routing scheme in WSANs, which illustrate that HTDM routing scheme is feasible. Figure 6 gives that experiment screenshot of creating an actor-actor routing path according to the traditional routing scheme in experimental scene (1). It can be seen from Fig. 6: if the system only creates a single routing path, namely the shortest routing path, in this case, the network energy consumption is given in Fig. 7. The energy consumption in the area passed by the routing path is very high, and the energy consumption in other area is low, which can cause energy consumption in the network unbalanced. At the same time, it can been seen from the following experiment that the network throughput of sensor node is small when the system creates a single routing path among actors, due to the huge gap of transmission capacity between actors and sensor nodes. Thus, the network throughput is limited and the data transmission time is long.

The experiment screenshots of three routing paths created by HTDM routing scheme from actor set 2 (AS2) to actor set (AS3) in scene (1) is given in Fig. 8. Data packet can simultaneously be routed to destination

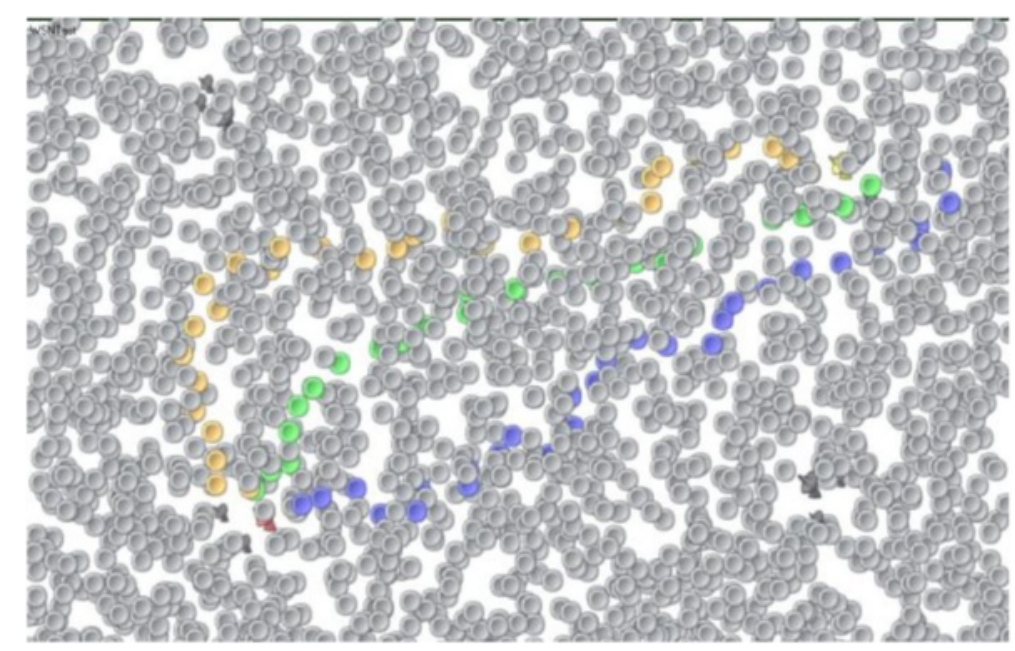

Fig. 8 Experimental screenshot of multi-route path (scene 1) 


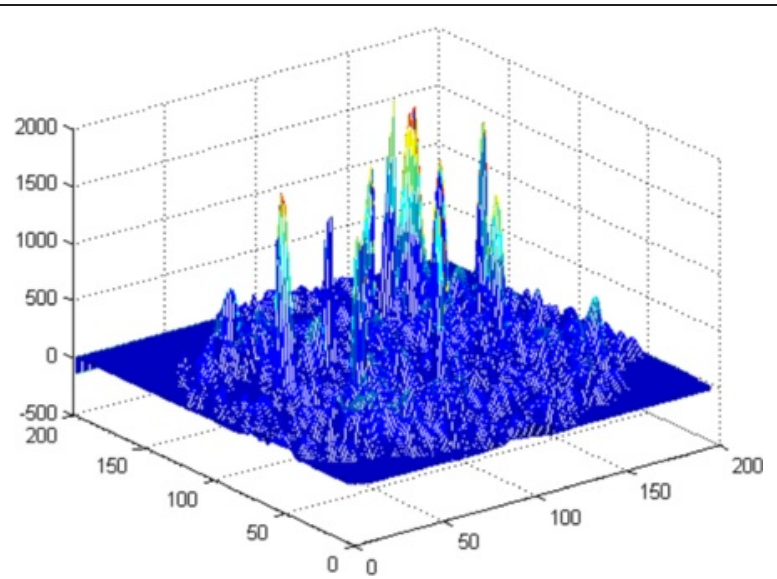

Fig. 9 The energy consumption in the multi-route path strategy (scene 1)

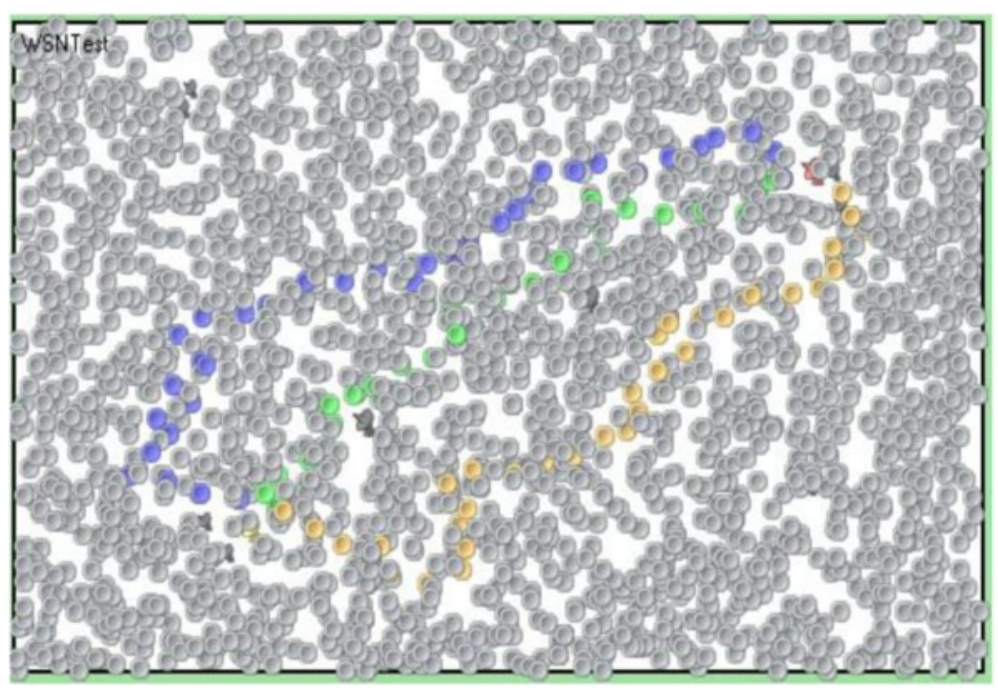

Fig. 10 Experimental screenshot of multi-route path establishment (scene 2)

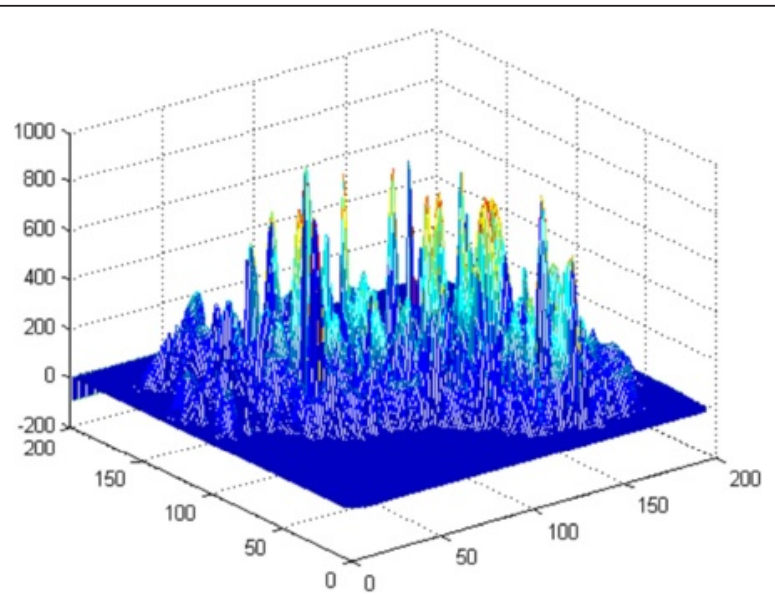

Fig. 11 The energy consumption in the multi-route path strategy (scene 2) 


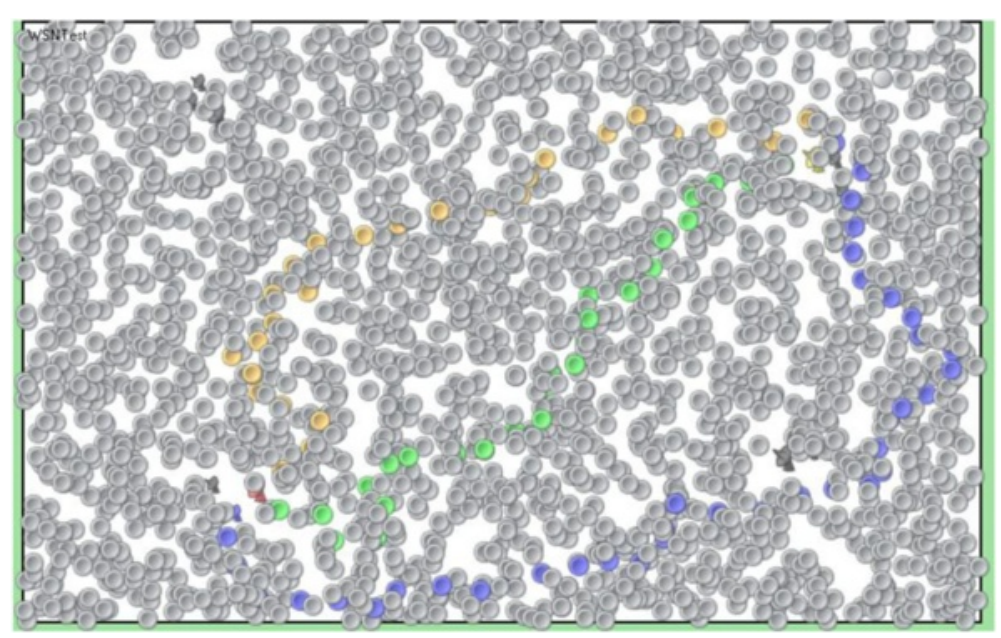

Fig. 12 Experimental screenshot of EHTDM routing scheme (scene 1)

actor by multiple routing in HTDM scheme. The bottleneck problem can be alleviated in actor-sensor-actor communication (ASAc), and the communication capacity among actors can be improved several times. On the other hand, multiple routing paths are running at the same time, the network energy consumption is relatively balanced, such as Fig. 9 .

The experiment screenshots of three routing paths created by HTDM routing scheme from actor set 1 (AS1) to actor set 4 (AS4) in scene (2) is given in Fig. 10. The energy consumption can be shown in Fig. 11. Multiple routing are established in the HTDM routing scheme to fill the gap of communication capability in actor-sensor-actor communication based on the shortest routing approach. However, this scheme can cause the energy consumption in the area of multiple paths pass by is much higher than other areas, which affects the network lifetime. The balanced energy consumption for whole network does not take into account in HTDM scheme. Therefore, EHTDM routing scheme builds routing path through selecting the path of the largest residual energy, which can bypass the node whose energy is not rich, so the scheme can balance the network energy consumption in whole network and prolong network lifetime. When multiple routing paths are need to be established from actor set 2 to actor set 3, in EHTDM scheme, the routing path can be established from actor set 2 to actor set 4 (see the bottom of routing path from Fig. 12), then from actor 4 to actors 3 . Although this routing length is longer than the routing which is routed directly from actor set 2 to actor set 3 , the more residual energy can be full used on those areas whose node's residual energy is rich. So, the energy consumption is more balance in whole network which can be seen from Fig. 13.

\subsection{The comparison of throughput}

Before analysis, the performance of different schemes, the reason which the performance of HTDM scheme is better than previous multi-path routing scheme [42] is shown in this part. The HTDM routing scheme and the multi-path routing scheme are given in Figs. 14 and 15, respectively. In HTDM routing scheme, the source and terminal of the route are composed by two parts: source node group with multiple actors and receiver node group. The communication capacity of actor is bigger than sensor node; the group composed with multiple actors can transmit data simultaneously. So, the throughput of multi-path routing scheme is big. But in multipath routing scheme [42], only a sensor node with low capacity is in source node and receiver node. Though

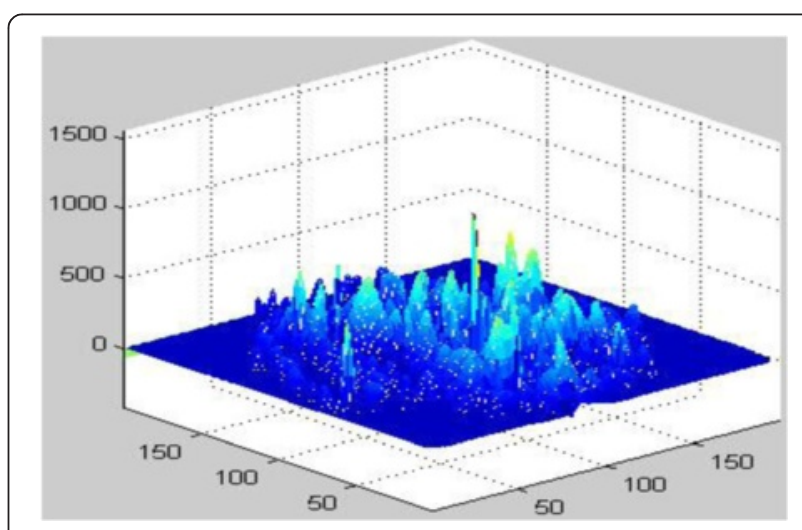

Fig. 13 The energy consumption of EHTDM routing scheme (scene 1) 


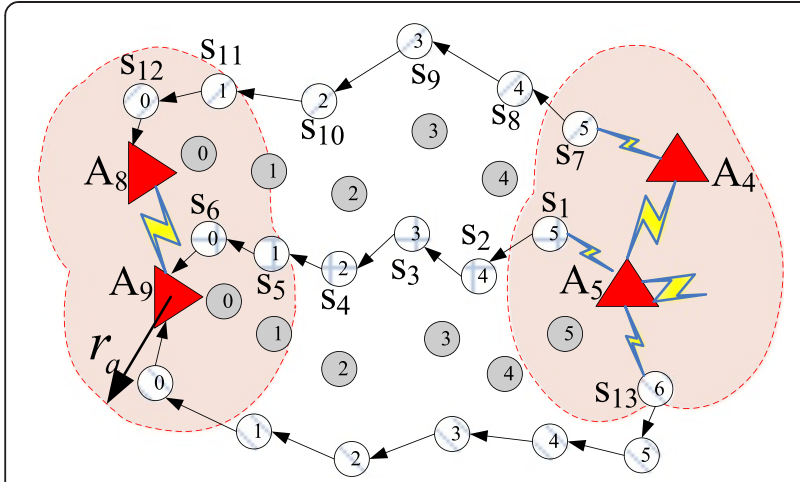

Fig. 14 The HTDM routing scheme

three routing paths are created, the capacity is determined by the sender capacity of source node or the receiver capacity of receiver node. Though the capacity of throughput is increased in this scheme compared to single routing path scheme, the increased capacity of throughput is limited. It is only bigger than the throughput of one routing path in HTDM routing scheme, but is smaller than the throughput of two routing paths in HTDM routing scheme. Multiple routing paths are created in HTDM routing scheme, so the performance of HTDM scheme is better than the previous multi-path routing scheme.

The experimental results of the throughput of HTDM routing scheme in network scenario 1 and 2 are given in Figs. 16 and 17, respectively. For network scenario 1, the routing path is from actor set 2 (AS2) to actor set (AS3), and in network scenario 2, the routing path is from actor set 1 (AS1) to actor set (AS4).

The proposed HTDM routing scheme is a kind of real multiple routing scheme; multiple routing paths are created among actors, which make up the difference of communication capacity between actors and

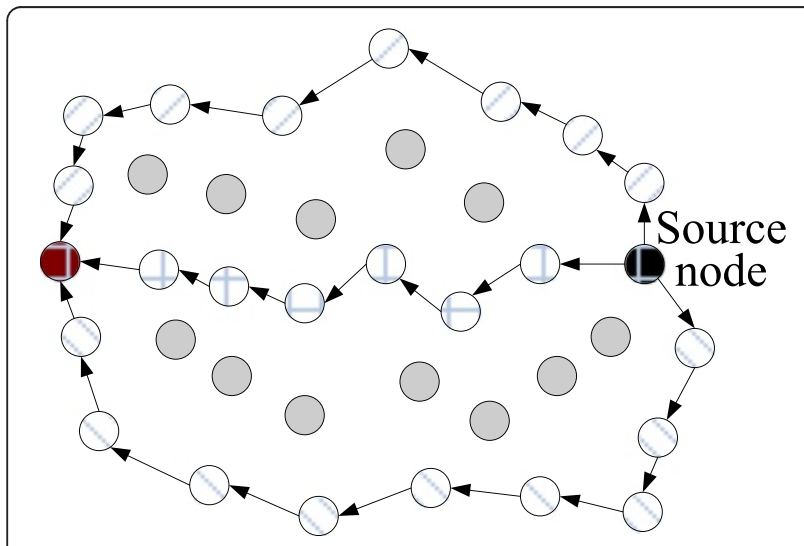

Fig. 15 The multi-path routing scheme

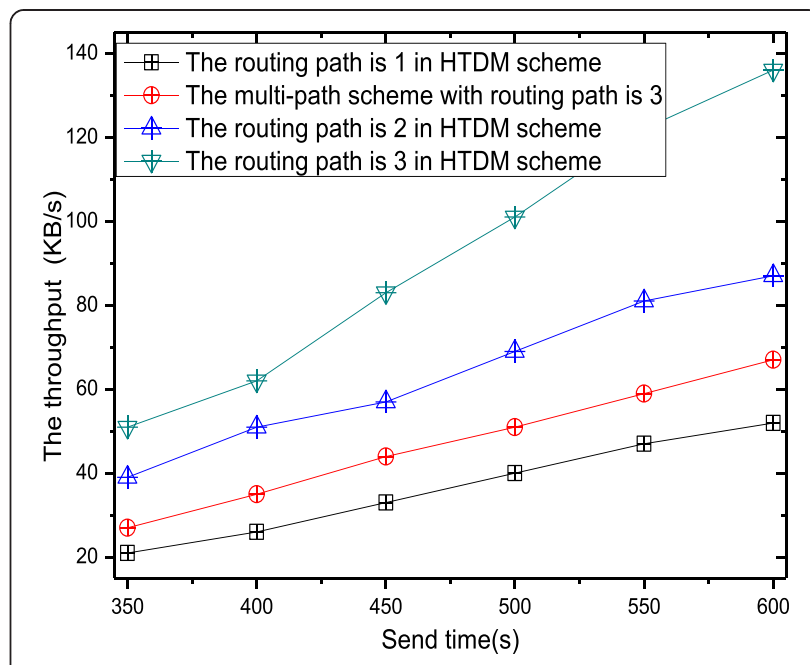

Fig. 16 The throughput of HTDM routing scheme vs multi-path scheme (scene 1)

sensor nodes. It can be seen from Figs. 16 and 17 that the throughput in the HTDM routing scheme is almost proportional to the number of creating routing paths. Therefore, the number of created routing paths can be identified according to the practical demand, which can offset the difference of communication ability between actors and sensor nodes. From experimental results, the throughput of multi-path routing scheme is smaller than that of HTDM scheme which creates two routing paths, and it is much smaller than that of HTDM scheme which creates three routing paths. Those results show HTDM scheme has a better throughput.

Similarly, Figs. 18 and 19 show the experiment results of the throughput of EHTDM routing scheme in

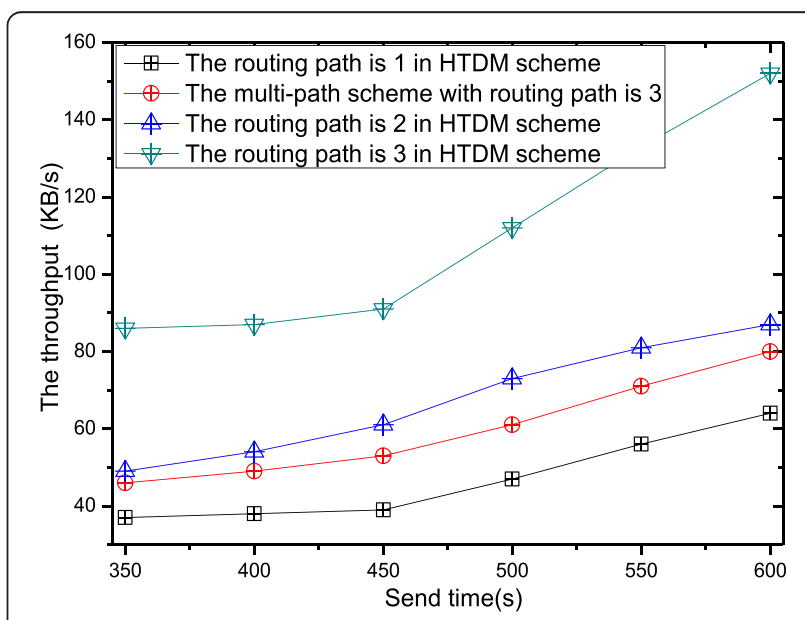

Fig. 17 The throughput of HTDM routing scheme vs multi-path scheme (scene 2) 


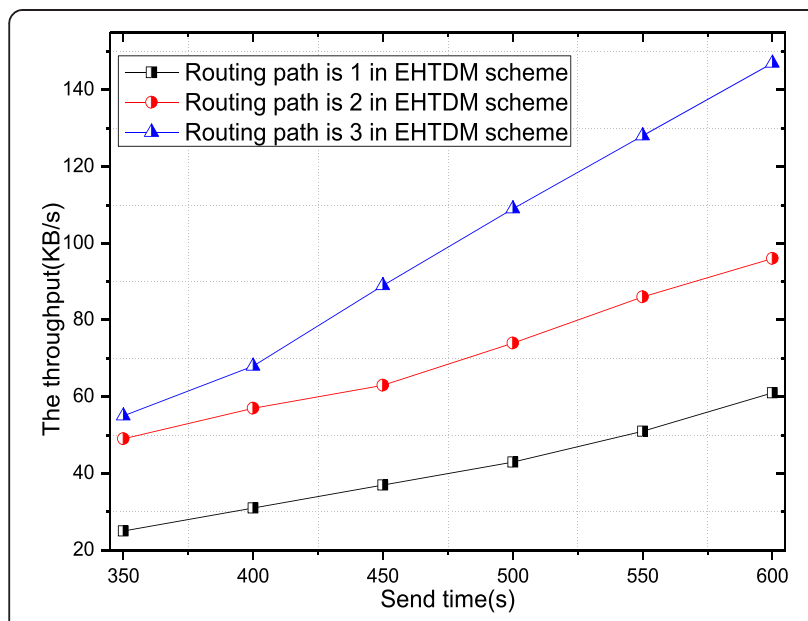

Fig. 18 The throughput of EHTDM routing scheme (scene 1)

network scenario 1 and 2. Because EHTDM routing scheme can only improve the capacity of balancing the energy consumption, the experimental results are almost the same as the above.

Figures 20 and 21 show the comparison of the throughput between HTDM and EHTDM routing scheme. Experiment setting is the number of creating multiple routing paths are three. The routing path is from actor set 2 (AS2) to actor set (AS3) in network scenario 1 , and the routing path is from actor set 1 (AS1) to actor set (AS4) in network scenario 2. It can be seen from the experimental results that the throughput in HTDM scheme is higher than EHTDM scheme. Because HTDM scheme creates multiple routing paths based on the principle of shortest path way, the routing path is shorter. But for EHTDM scheme, in order to balance the energy consumption,

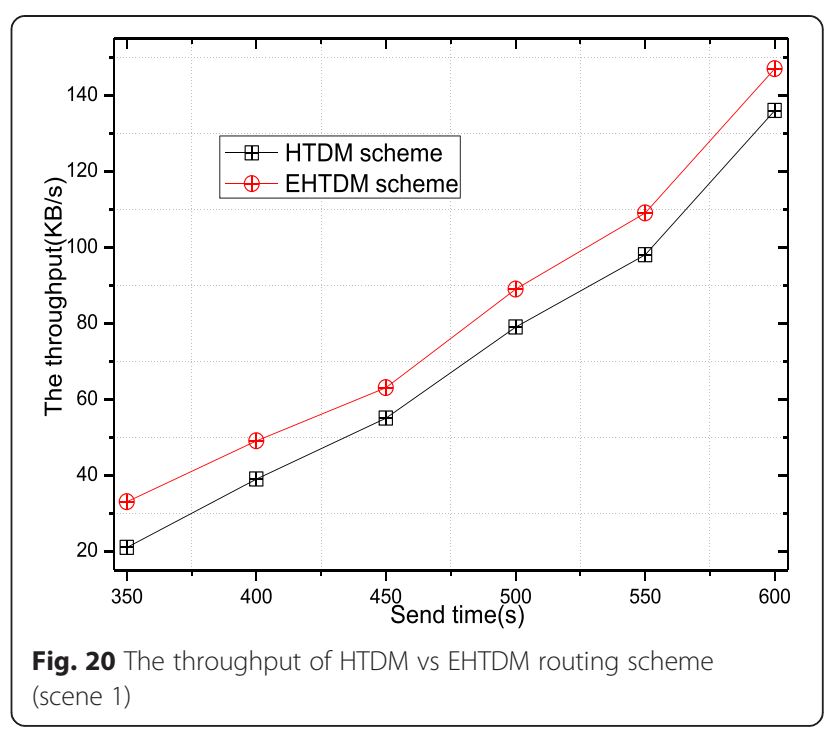

the path often needs to pass the area with high energy consumption to transmit data, so the routing path is longer, which lead to more nodes need to be passed by for transmitting data to the target node (see Section 5.4), and more time is required to complete the routing. Although the throughput in EHTDM scheme is lower than HTDM scheme, it can balance the network energy consumption and prolong network lifetime (see Section 5.5).

\subsection{The comparison of delay}

The delay of HTDM routing scheme vs multi-path routing scheme in different network scenarios is given in Figs. 22 and 23. The parameters in the experiment are the same as the previous experiments. The delay refers to the requirement time for transmitting a certain

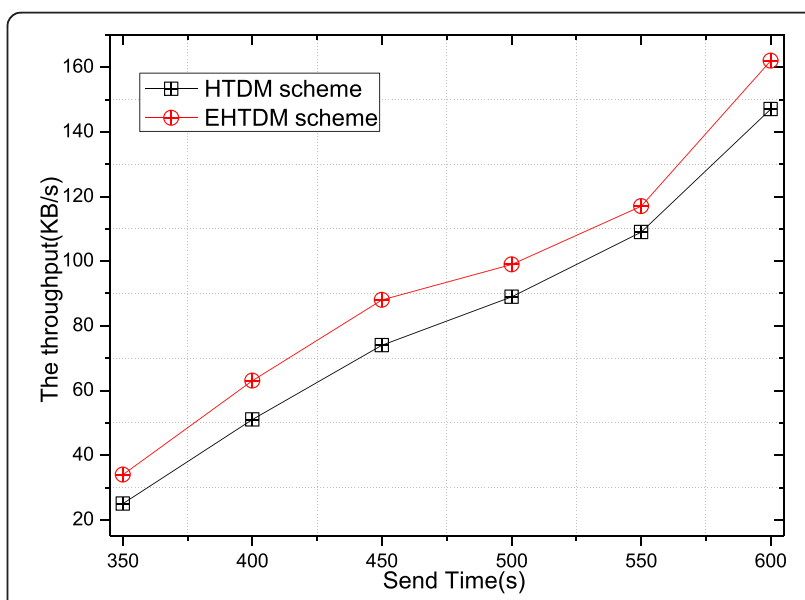

Fig. 21 The throughput of HTDM vs EHTDM routing scheme (scene 2)

Fig. 19 The throughput of EHTDM routing scheme (scene 2) 


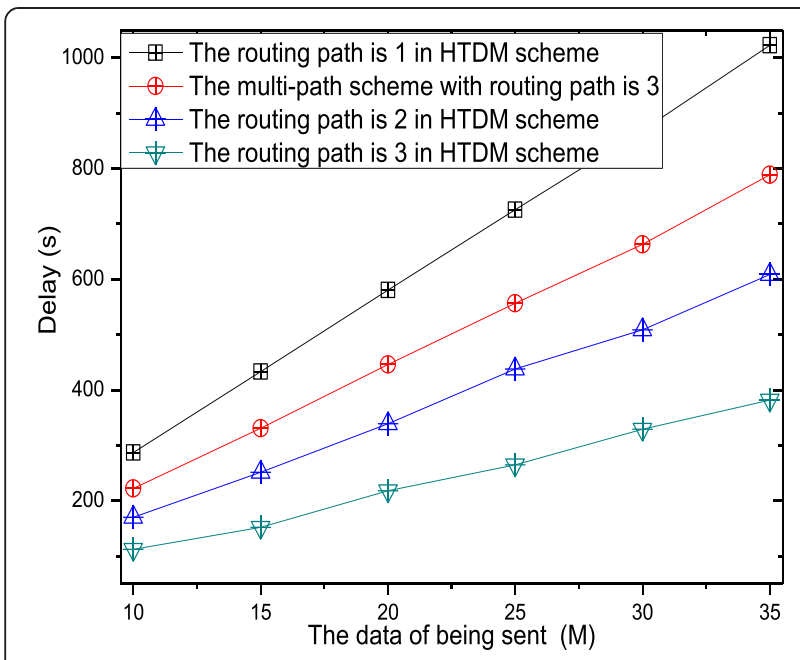

Fig. 22 The delay of HTDM routing scheme vs multi-path routing scheme (scene 1)

amount of data from one actor to another actor. The experimental results confirm the effectiveness of our proposed strategy.

The delay of EHTDM routing scheme in different network scenarios are given in Figs. 24 and 25. Similarly, EHTDM routing scheme can also reduce the requirement time for transmitting data by creating multiple routing paths. However, the requirement time in EHTDM scheme is relatively longer, this is the cost to make the network energy consumption balanced.

5.4 The experiment of the requirement hops for routing The number of total required hop count for routing in HTDM scheme and EHTDM scheme are given in

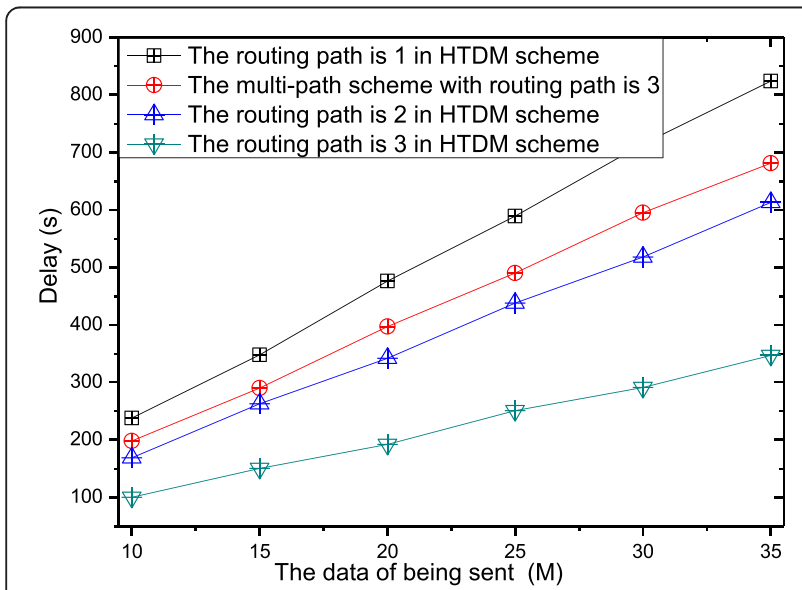

Fig. 23 The delay of HTDM routing scheme vs multi-path routing scheme (scene 2)

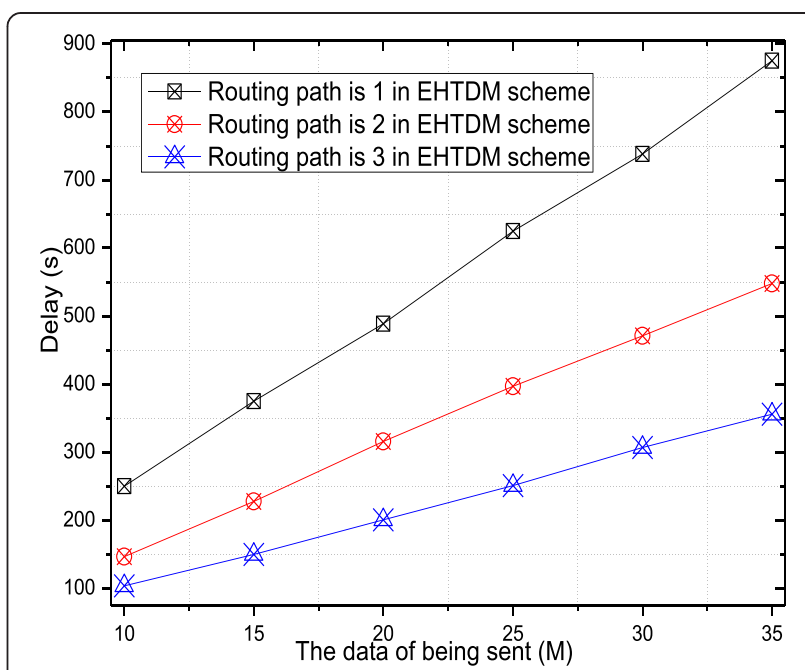

Fig. 24 The delay of EHTDM routing scheme (scene 1)

Figs. 26 and 27. It can be seen from the experimental results: (1) The number of required hop count for routing is proportional to the number of creating routing paths. (2) The number of required hop count in EHTDM scheme is greater than that of HTDM scheme's, the more the number of creating routing paths are, the more its routing hop counts are. This shows that to balance the energy consumption, the data packets are routed around the farther nodes with the more residual energy in EHTDM scheme. Therefore, the routing needs more hop count to route data packets, and the routing path consumes more energy, which can make full use of the residual energy of the nodes and prolong the network lifetime (see the next section).

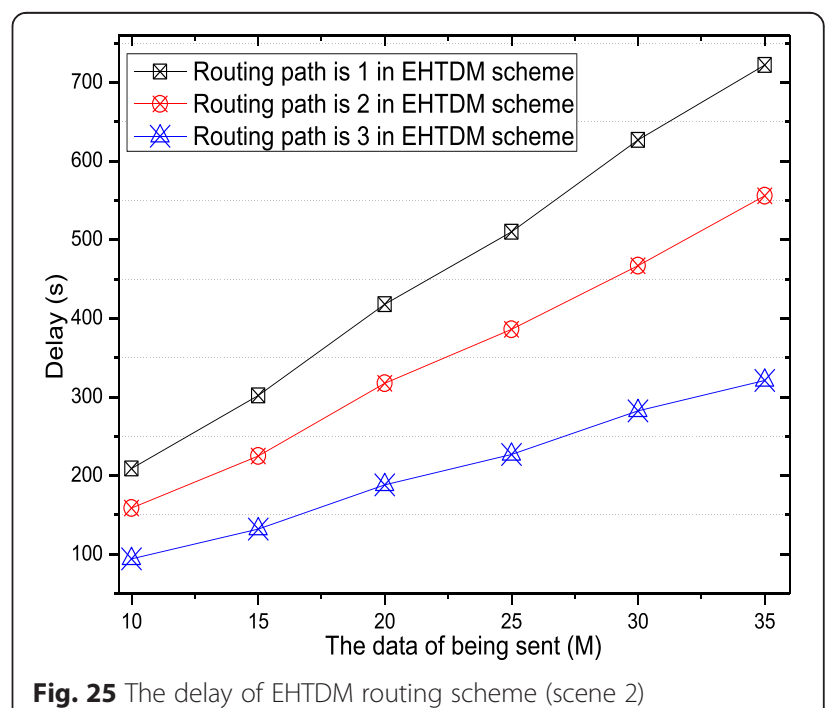




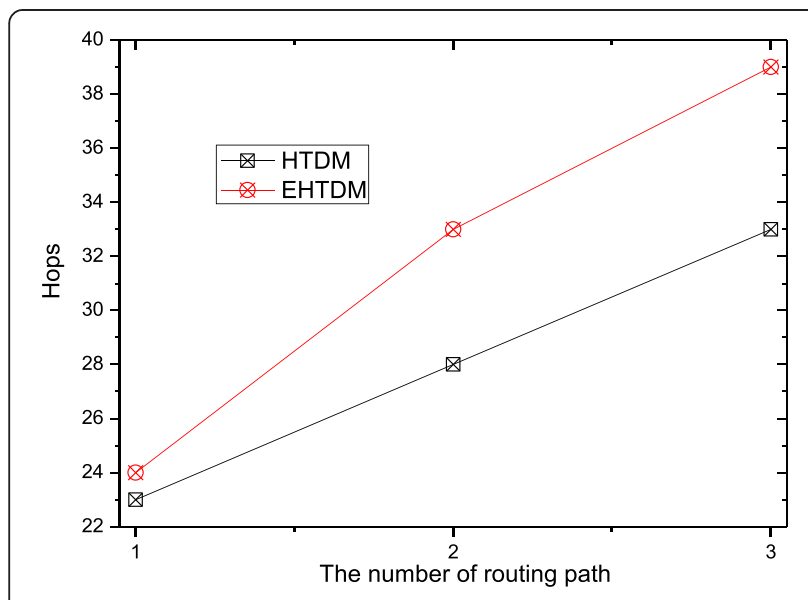

Fig. 26 The total hops of routing (scene 1)

\subsection{The comparison of network lifetime}

Figures 28 and 29, respectively, give the network lifetime of multi-path routing scheme, HTDM and EHTDM routing scheme in network scene (1) and (2). As it can be seen from the results of the experiment, EHTDM routing scheme can balance the network energy consumption, so the network lifetime in EHTDM routing scheme is better than HTDM scheme. It shows the effectiveness of the proposed scheme. For multi-path routing scheme, due to its energy consumption is almost equal to HTDM scheme's, the lifetime of multi-path routing scheme is almost equal to HTDM scheme's.

\section{Conclusion}

In WSANs, the communication among actors in different partition can be completed through relaying the data to the destination actor by sensor node, but the difference of throughput capacity between sensor

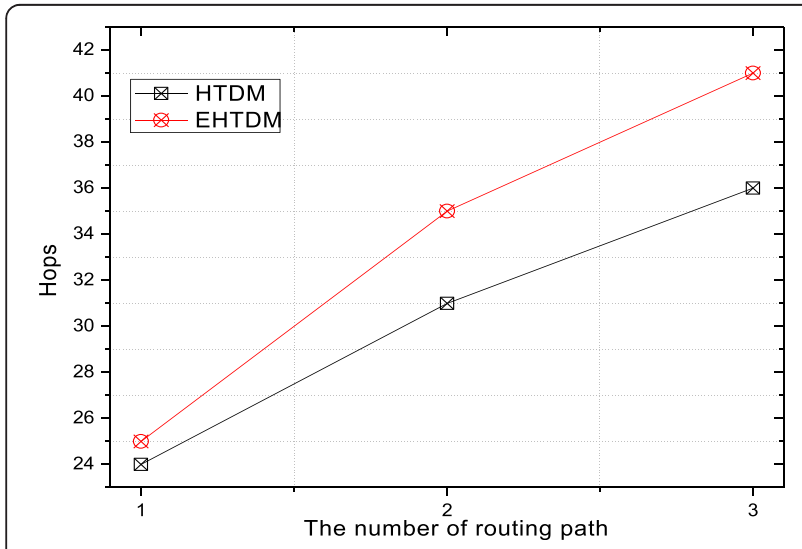

Fig. 27 The total hops of routing (scene 2)

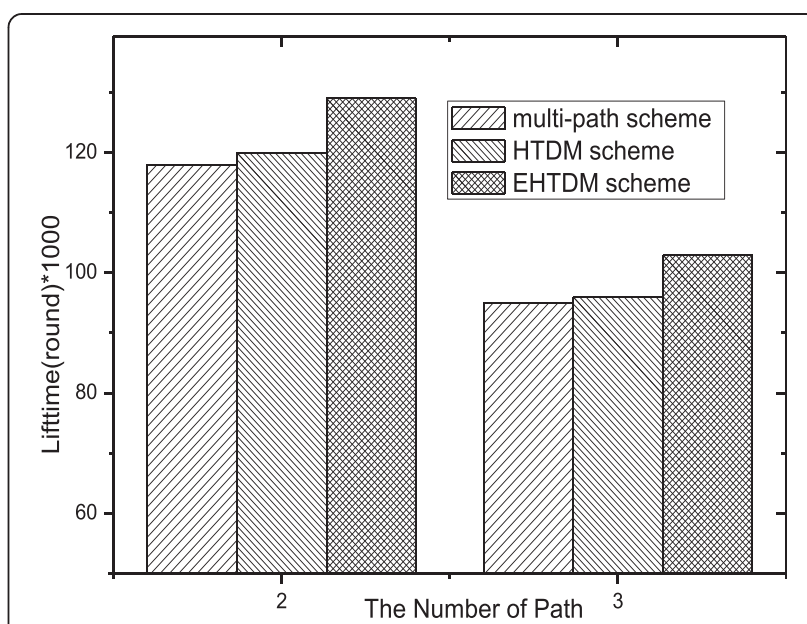

Fig. 28 The lifetime in difference scheme (scene 1)

nodes and actors is huge, which affects the throughput of the network. In this paper, a novel highthroughput disjoint multi-path (HTDM) routing scheme is proposed to fill the gap. In the thesis, the routing protocol of HTDM scheme is introduced in detail. It has proved that multiple routing paths are established among actor sets in HTDM scheme, thus bridging the gap among actor-sensor-actor communication (ASAc). Then, an EHTDM scheme is proposed to balance the energy consumption and prolong network lifetime. The results of the experiment show that the proposed scheme can significantly improve the network capacity and reduce the time for transferring data. In this paper, the HTDM scheme did not involve the safety problems; we will study the secure routing scheme in the future $[22,32,52]$.

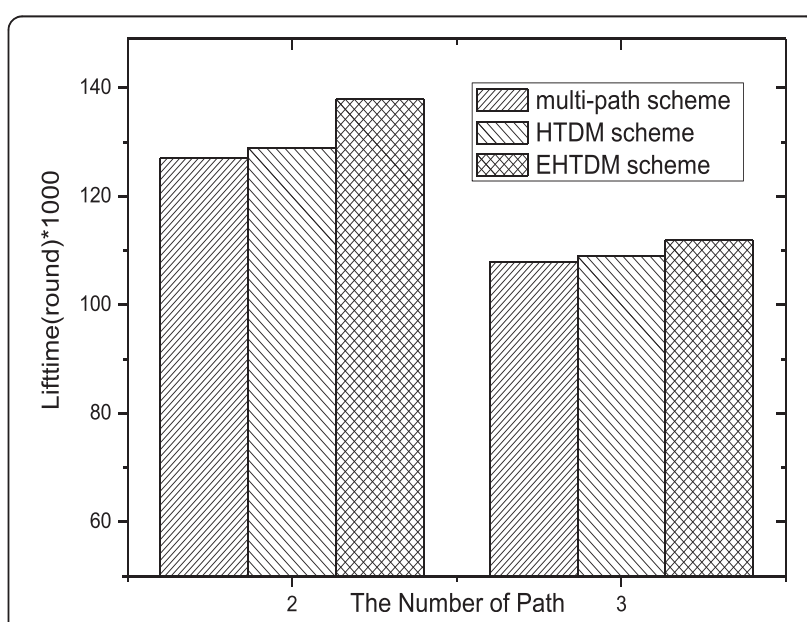

Fig. 29 The lifetime in difference scheme (scene 2) 


\section{Competing interests}

The authors declare that they have no competing interests.

\section{Acknowledgements}

Supported by the National Natural Science Foundation of China (61379110, 61073104, 61472450, and 61272150) and the National Basic Research Program of China (973 Program) (2014CB046305).

\section{Received: 30 September 2015 Accepted: 18 November 2015 Published online: 08 December 2015}

\section{References}

1. K Selvaradjou, $\mathrm{N}$ Handigol, AA Franklin et al., Energy-efficient directional routing between partitioned actors in wireless sensor and actor networks. IET Commun. 4(1), 102-115 (2010)

2. Y Zhou, ECH Ngai, MR Lyu, et al. POWER-SPEED: a power-controlled real-time data transport protocol for wireless sensor-actuator networks. Wireless Communications and Networking Conference, 2007. WCNC 2007, 2007: 3736-3740.

3. Y Liu, A Liu, Z Chen, Analysis and improvement of send-and-wait automatic repeat-reQuest protocols for wireless sensor networks. Wirel. Pers. Commun. 81(3), 923-959 (2015)

4. M Li et al., A survey on topology control in wireless sensor networks: taxonomy, comparative study, and open issues. Proc. IEEE 101(12), 2538-2557 (2013)

5. N Chilamkurti, et al. Cross-layer support for energy efficient routing in wireless sensor networks, Journal of Sensors 2009

6. Y Yao et al., EDAL: an energy-efficient, delay-aware, and lifetime-balancing data collection protocol for heterogeneous wireless sensor networks. IEEE/ACM Trans. Networking 23(3), 810-823 (2015)

7. Y Hu, A Liu, An efficient heuristic subtraction deployment strategy to guarantee quality of event detection for WSNs. Comput. J. 58(8), 1747-1762 (2015)

8. K Han, J Luo, Y Liu et al., Algorithm design for data communications in duty-cycled wireless sensor networks: a survey. IEEE Commun. Mag. 51(7), 107-113 (2013)

9. Z Z Sheng et al., A survey on the ietf protocol suite for the internet of things: standards, challenges, and opportunities. IEEE Wirel. Commun. 20(6), 91-98 (2013)

10. Y Xiao et al., Tight performance bounds of multihop fair access for MAC protocols in wireless sensor networks and underwater sensor networks. IEEE Trans. Mob. Comput. 11(10), 1538-1554 (2012)

11. Y Zeng et al., Directional routing and scheduling for green vehicular delay tolerant networks. Wirel. Netw 19(2), 161-173 (2013)

12. L Jiang, A Liu, Y Hu, Z Chen, Lifetime maximization through dynamic ring-based routing scheme for correlated data collecting in WSNs. Computers \& Electrical Engineering 41, 191-215 (2015)

13. X. Liu, et al. Compressed data aggregation for energy efficient wireless sensor networks. SECON 2011: 46-54

14. S Sengupta et al., An Evolutionary multiobjective sleep-scheduling scheme for differentiated coverage in wireless sensor networks. IEEE Transactions on Systems, Man, and Cybernetics, Part C 42(6), 1093-1102 (2012)

15. G Wei et al., Prediction-based data aggregation in wireless sensor networks: combining grey model and Kalman filter. Comput. Commun. 34(6), 793-802 (2011)

16. X Liu et al., CDC: compressive data collection for wireless sensor networks. IEEE Transactions on Parallel \& Distributed Systems 26(8), 2188-2197 (2015)

17. Y Song et al., A biology-based algorithm to minimal exposure problem of wireless sensor networks. IEEE Trans. Netw. Serv. Manag. 11(3), 417-430 (2014)

18. Y Liu et al., Multi-layer clustering routing algorithm for wireless vehicular sensor networks. IET Commun. 4(7), 810-816 (2010)

19. $\mathrm{XXu}$, et al. Hierarchical data aggregation using compressive densing (HDACS) in WSNs. ACM Transactions on Sensor Networks (TOSN), 11(3) (2015).

20. M Bhuiyan, G Wang, A Vasilakos, Local area prediction-based mobile target tracking in wireless sensor networks. IEEE Trans. Computers 64(7), 1968-1982 (2015)

21. Y Guo, Z Xu, C Chen et al., DGR: dynamic gradient-based routing protocol for unbalanced and persistent data transmission in wireless sensor and actor networks. Journal of Zhejiang University SCIENCE 12(4), 273-279 (2011)
22. Y Liu, A Liu, S He, A novel joint logging and migrating traceback scheme for achieving low storage requirement and long lifetime in WSNs. AEU-International Journal of Electronics and Communications 69(10), 1464-1482 (2015)

23. C Busch, R Kannan, AV Vasilakos, Approximating congestion + dilation in networks via "quality of routing" games. IEEE Trans. Comput. 61(9), 1270-1283 (2012)

24. P Li et al., Reliable multicast with pipelined network coding using opportunistic feeding and routing. IEEE Transactions on Parallel Distributed Systems 25(12), 3264-3273 (2014)

25. A Dvir et al., Backpressure-based routing protocol for DTNs. ACM SIGCOMM Computer Communication Review 41(4), 405-406 (2011)

26. A.Vasilakos, et al., Delay Tolerant Networks: Protocols and Applications. CRC Press, 2012

27. M Dong et al., Joint Optimization of Lifetime and Transport Delay under Reliability Constraint Wireless Sensor Networks, accepted by IEEE Transactions on Parallel and Distributed Systems. DOI: 10.1109/TPDS.2015. 2388482

28. A Liu, X Jin, G Cui et al., Deployment guidelines for achieving maximum lifetime and avoiding energy holes in sensor network. Inf. Sci. 230, 197-226 (2013)

29. L Liu et al., Physarum optimization: a biology-inspired algorithm for the Steiner tree problem in networks. IEEE Trans. Comput. 64(3), 819-832 (2015)

30. T Meng, TF Wu, Z Yang, et al. Spatial reusability-aware routing in multihop wireless networks. IEEE Transactions on Computers, doi: 10.1109/TC. 2015.2417543

31. Z Liang et al., Context-aware middleware for multimedia services in heterogeneous networks. IEEE Intell. Syst. 25(2), 40-47 (2010)

32. M Dong, X Liu, Z Qian et al., QoE-ensured price competition model for emerging mobile networks. IEEE Wirel. Commun. 22(4), 50-57 (2015)

33. J Long, A Liu, M Dong et al., An energy-efficient and sink-location privacy enhanced scheme for WSNs through ring based routing. Journal of Parallel and Distributed Computing 81, 47-65 (2015)

34. G Acampora et al., Interoperable and Adaptive Fuzzy Services for Ambient Intelligence Applications ACM Transactions on Autonomous and Adaptive Systems (TAAS) 5 (2), 8, 2010

35. J Zhou et al., Secure and privacy preserving protocol for cloud-based vehicular DTNs. IEEE Transactions on Information Forensics and Security 10(6), 1299-1314 (2015)

36. ZM Fadlullah, T Taleb, AV Vasilakos et al., DTRAB: combating against attacks on encrypted protocols through traffic-feature analysis. IEEE/ACM Transactions on Networking (TON) 18(4), 1234-1247 (2010)

37. $\mathrm{Y} \mathrm{Hu}, \mathrm{M}$ Dong, $\mathrm{K}$ Ota, et al. Mobile target detection in wireless sensor networks with adjustable sensing frequency. IEEE System Journal, doi: 10. 1109/JSYST.2014.2308391

38. Q Jing, AV Vasilakos, J Wan et al., Security of the Internet of Things: perspectives and challenges. Wirel. Netw 20(8), 2481-2501 (2014)

39. X Liu, M Dong, K Ota, P Hung, A Liu. Service pricing decision in cyberphysical systems: insights from game theory. IEEE Transactions on Services Computing. doi: 10.1109/TSC.2015.2449314.

40. Z Yan, ZP Zhang, AV Vasilakos, A survey on trust management for Internet of Things. J. Network and Computer Applications 42, 120-134 (2014)

41. J Gui, M Ahmadi, F Tong, Dynamically constructing and maintaining virtual access points in a macro cell with selfish nodes. J. Syst. Softw. 108, 1-22 (2015)

42. BY Li, PJ Chuang, Geographic energy-aware non-interfering multipath routing for multimedia transmission in wireless sensor networks. Inf. Sci. 249, 24-37 (2013)

43. J Gui, Z Zeng, Joint network lifetime and delay optimization for topology control in heterogeneous wireless multi-hop networks. Comput. Commun. 59, 24-36 (2015)

44. Mi Akbaş, D Turgut, Lightweight routing with dynamic interests in wireless sensor and actor networks. Ad Hoc Netw. 11(8), 2313-2328 (2013)

45. $\mathrm{S} \mathrm{He}, \mathrm{X} \mathrm{Li}$, J Chen et al., EMD: energy-efficient P2P message dissemination in delay-tolerant wireless sensor and actor networks. IEEE Journal on Selected Areas in Communications 31(9), 75-84 (2013)

46. T Melodia, D Pompili, VC Gungor et al., Communication and coordination in wireless sensor and actor networks. IEEE Trans. Mob. Comput. 6(10), 1116-1129 (2007)

47. J Cho, J Lee, T Kwon et al., Directional antenna at sink (DAaS) to prolong network lifetime in wireless sensor networks Wireless Conference 2006-Enabling Technologies for Wireless Multimedia Communications (European Wireless), 12th European. VDE, 2006, pp. 1-5 
48. C. Y. Wan, S. B. Eisenman, A. T. Campbell, et al. Siphon: overload traffic management using multi-radio virtual sinks in sensor networks. Proceedings of the 3rd international conference on Embedded networked sensor systems. ACM, 2005: 116-129.

49. K Ota, M Dong, Z Cheng et al., ORACLE: mobility control in wireless sensor and actor networks. Comput. Commun. 35(9), 1029-1037 (2012)

50. J. Long, A. He, A. Liu, et al. Adaptive sensing with reliable guarantee under white gaussian noise channels of sensor networks. Journal of Sensors, 2015, (2015)

51. The OMNET++ simulator. [Online] Available: http://www.omnetpp.org/. Accessed 10, 2015

52. U Bodin, K Wolosz, Proportional throughput differentiation with cognitive load-control on WSN channels. EURASIP J. Wirel. Commun. Netw. 2015(1), 1-14 (2015)

Submit your manuscript to a SpringerOpen ${ }^{\odot}$ journal and benefit from:

- Convenient online submission

- Rigorous peer review

- Immediate publication on acceptance

- Open access: articles freely available online

- High visibility within the field

- Retaining the copyright to your article

Submit your next manuscript at $>$ springeropen.com 